\title{
A Body Mass Index-Based Cross-Classification Approach for the Assessment of Prognostic Factors in Chronic Kidney Disease Progression
}

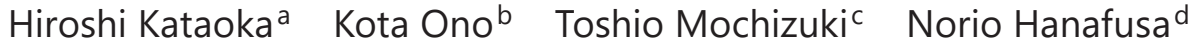 \\ Enyu Imai ${ }^{\text {Akira Hishida }}{ }^{f}$ Kosaku Nitta ${ }^{a}$ \\ a Department of Internal Medicine, Tokyo Women's Medical University, Tokyo, Japan; \\ ${ }^{b}$ Medical Innovation Center, Hokkaido University Hospital, Hokkaido, Japan; \\ ${ }^{c}$ Clinical Research Division for Polycystic Kidney Disease, Department of Medicine, \\ Kidney Center, Tokyo Women's Medical University, Tokyo, Japan; d Department of \\ Blood Purification, Kidney Center, Tokyo Women's Medical University, Tokyo, Japan; \\ e Nakayamadera Imai Clinic, Takarazuka, Japan; fYaizu City Hospital, Yaizu, Japan
}

\section{Keywords}

Cross classification - Subgroup analysis - Data disaggregation - Body mass index · Chronic kidney disease $\cdot$ Pulsatile stress · CKD-JAC

\begin{abstract}
Background/Aims: Cross-classification analyses are rarely reported. We investigated the prognostic factors for chronic kidney disease (CKD) progression using a body mass index (BMI)-based cross-classification approach. Methods: Patients' renal outcome ( $\geq 50 \%$ decline in the estimated glomerular filtration rate or end-stage renal disease) in each subcohort was examined. Results: The number of prognostic factors identified in the multivariate Cox analysis was smaller in the "BMI $\geq 25$, female" and CKD stage 3 subcohorts than in other subcohorts. Prognostic factors identified in the "BMI $\geq 25$, CKD stage 3 " subcohort only comprised albuminuria and male sex, and those in the "BMI $\geq 25$, female" subcohort only comprised albuminuria, hyperphosphatemia, and anemia. Albuminuria, kidney impairment, male sex, hyperphosphatemia, anemia, and increased pulse pressure $\times$ heart rate product $(\mathrm{PP} \times \mathrm{HR}$; pulsatile stress) were stable renal prognostic factors in almost all subcohorts. On the other hand, the prognostic value of increased BMI, younger age, hypoalbuminemia, increased intact parathyroid hormone, and decreased estimated 24-h urinary potassium excretion (e24hUK) differed according to subcohort. BMI was positively associated with CKD progression in the "BMI $\geq 25$, age $\geq 65$ years" and "BMI $\geq 25$, CKD stages $4-5$ " subcohorts, whereas it was negatively associated with CKD progression in the "BMI $<25$, diabetes mellitus" subcohort. PP $\times$ HR was independently associated with CKD progression in the "BMI <25, CKD stage 3" subcohort,
\end{abstract}


which had relatively few identified renal prognostic factors. Decreased e24hUK was a renal prognostic factor for CKD progression in the "BMI <25, CKD stages 4-5" subcohort, while no significant factors were observed in the "BMI $\geq 25$, CKD stages 4-5" subcohort. Conclusion: A $\mathrm{BMI}$-based cross-classification approach, which provides more comprehensive findings than that in previous approaches, is expected to be an effective method for evaluating renal prognostic factors in patients with CKD who are affected by multiple risk factors.

(C) 2019 The Author(s)

Published by S. Karger AG, Basel

\section{Introduction}

In clinical studies, subgroup analyses are not usually recommended because of the risk for false-positive or false-negative results. Furthermore, although conventional subgroup analyses commonly involve stratification analyses, which assess the influence of a single attribute, cross-classification analyses, which simultaneously assess the influence of multiple attributes, have rarely been reported. However, investigations utilizing a data disaggregation or cross-classification approach in medical settings with diverse patients can produce results closely reflecting the real clinical world.

Body mass index (BMI $\left[\mathrm{kg} / \mathrm{m}^{2}\right]$ ) has drawn attention as a prognostic factor for chronic kidney disease (CKD) [1]. However, the effect of excessive weight and obesity on CKD progression is controversial. Some studies on immunoglobulin A nephropathy have reported obesity as a predictor of CKD progression [2,3], while a recent study has reported that underweight status is a risk factor for renal function deterioration [4]. The hazard or OR for BMI as a renal prognostic factor adopts a J-shaped or U-shaped curve [5, 6]. Given this nonlinearity, it may be more effective to use BMI as an attribute-determination factor, rather than as a prognostic factor, in cross-classification analyses. The present study is the first to conduct a detailed investigation of the prognostic factors for CKD progression using a BMI-based crossclassification approach.

\section{Materials and Methods}

\section{Study Design}

This study utilized data from the CKD Japan Cohort study, a large-scale, multicenter, prospective, cohort study in Japan (UMIN clinical trial registry number: UMIN000020038) [7,8]. The study protocol was approved by the Ethics Committee of each participating medical institution (Tokyo Women's Medical University: \#1106). The study was conducted in accordance with the Declaration of Helsinki, 1975 (as revised in 2005) and in compliance with the current regulations. A total of 2,678 patients from 17 nephrology centers were enrolled, and the primary outcome was defined as a $\geq 50 \%$ decline in the estimated glomerular filtration rate (eGFR) relative to baseline or end-stage renal disease (ESRD). We initially examined the prognostic factors of CKD in the total cohort and subsequently divided the patients according to BMI ( $<25 \mathrm{vs.} \geq 25$ ). The final subcohorts were formed by multiplying each of 4 conventional attributes (age, sex, CKD stage, and diabetes mellitus [DM]) with the BMI classification, which resulted in a total of 16 subcohorts for the investigation of CKD progression.

\section{Inclusion Criteria}

A total of 3,087 patients from 17 nephrology centers were recruited between April 2007 and March 2013. The following inclusion criteria were used at screening: (1) Japanese patients living in Japan; (2) age 20-75 years; and (3) a broad spectrum of CKD, defined as an eGFR of 10-59 mL/min $/ 1.73 \mathrm{~m}^{2}$. The following patients were excluded from participation: (1) patients with polycystic kidney disease, human immunodeficiency virus infection, liver cirrhosis, active cancer, and patients who had received cancer treatment within 


\section{Kidney \\ Blood Pressure Research}

\begin{tabular}{l|l}
\hline Kidney Blood Press Res 2019:44:362-383 \\
\hline DOI: 10.1159/000501021 & $\begin{array}{l}\text { @ 2019 The Author(s). Published by S. Karger AG, Basel } \\
\text { www.karger.com/kbr }\end{array}$ \\
\hline
\end{tabular}

Kataoka et al.: BMI-Based Cross-Classification Approach for CKD

the past 2 years; (2) transplant recipients and patients who had previously been on long-term dialysis; (3) pregnant women; (4) patients who refused to provide written informed consent. eGFR was calculated by using a modified 3-variable equation for eGFR in Japanese patients [9]. Among recruited patients, 59 patients withdrew their consent, 27 fell under the exclusion criteria, 25 could not complete baseline data abstraction, 5 were excluded by the attending physician, 4 were lost to follow-up evaluation, and 1 patient died. Furthermore, we excluded 288 patients without baseline BMI data. After eliminating these patients from the registry, 2,678 participants were enrolled in the present study (online suppl. Appendix p 6: Appendix Fig. 1; for all online suppl. material, see www.karger.com/doi/10.1159/000501021).

\section{Procedures}

Eligible patients were evaluated at baseline. Age, sex, and smoking status were based on self-report. Information on sociodemographic information, family history, and past medical history, including hypertension, acute myocardial infarction, angina pectoris, congestive heart failure, peripheral arterial disease, cerebrovascular disease, and prescription of antihypertensive agents, including angiotensin-converting enzyme inhibitors, angiotensin receptor blockers, calcium channel blockers, diuretics, and $\beta$-blockers, statins, and antiplatelet agents, was collected from the medical records at each institution. Anthropometric measurements, blood pressure (BP), heart rate (HR), ankle-brachial index (ABI), and pulse wave velocity (PWV) were also collected at baseline. Each institution followed-up with the relevant participants every 6 months through a regular ambulatory visit, supplemented by a phone interview as needed. All patients were classified on the basis of CKD stage as described in the previous paper [8]. A history of cardiovascular disease was defined as a history of coronary heart disease, stroke, or heart failure.

\section{Data Collection}

Measurement of Hemodynamic Parameters

$\mathrm{BP}$ and HR were measured in outpatient clinics by using an automated sphygmomanometer after a 5 -min rest. BP in the right arm was measured 3 times at intervals of $1 \mathrm{~min}$, and the mean values were used for analyses. A mercury sphygmomanometer was used to measure the BP of patients who had frequent premature contractions, atrial fibrillation, or atrial flutter. Pulse pressure (PP) was calculated by subtracting diastolic BP from systolic BP.

Collection of Biological Samples and Measurements

Biological samples were collected annually and stored for the central laboratory. Whole blood, serum, and urine samples were collected for measurement of serum creatinine and cystatin C, HbA1c, intact parathyroid hormone (iPTH), and urinary albumin and creatinine levels. HbA1c was measured by the JDS method, and the value was converted to the A1C value measured by the NGSP method by adding $0.4 \%$ as determined by the Japanese Diabetes Society. Serum creatinine levels were measured enzymatically. Urine albumin and creatinine were assessed with the trypsin inhibitor activity and enzymatic methods, respectively. Urinary albumin excretion was expressed as the albumin to creatinine ratio (UACR). Total cholesterol concentrations were determined by an enzymatic method. Hemoglobin was measured by SLS-Hb method. The Tanaka formula [10] was used to estimate 24-h urinary sodium and potassium excretion from a casual urine specimen, taken at the baseline. Each clinical center measured serum creatinine at each visit. The correlations of serum creatinine concentrations measured at the respective clinical centers and central laboratories were examined, and the former concentrations corrected with the latter ones were used to address the interinstitutional heterogeneity of the data obtained. Furthermore, the functional relationship analysis estimating errors in the values measured at the respective clinical centers and central laboratories was conducted to make necessary corrections.

\section{Definition of Comorbidities and Primary Causes of CKD}

Hypertension was defined as systolic BP $\geq 140 \mathrm{~mm} \mathrm{Hg}$ or diastolic BP $\geq 90 \mathrm{~mm}$ Hg or taking an antihypertensive agent. Hyperuricemia was defined as serum UA level $\geq 6.0 \mathrm{mg} / \mathrm{dL}$ or taking an antihyperuricemic agent. Hypercholesterolemia was defined as serum TC level $\geq 220 \mathrm{mg} / \mathrm{dL}$ or serum LDL level $\geq 140 \mathrm{mg} /$ $\mathrm{dL}$ or taking an antidyslipidemic agent. Hypoalphalipoproteinemia was defined as serum HDL level $<40 \mathrm{mg} /$ $\mathrm{dL}$ or taking an antidyslipidemic agent. Hypertriglyceridemia was defined as serum TG level $\geq 150 \mathrm{mg} / \mathrm{dL}$ or taking an antidyslipidemic agent. DM was defined as $\mathrm{HbA} 1 \mathrm{C} \geq 6.5 \%$, a history of diagnosis with DM, or taking an antidiabetic agent. Diabetic nephropathy, chronic glomerulonephritis, and nephrosclerosis were defined by biopsy or clinical diagnosis by the doctor in charge. 


\section{Kidney \\ Blood Pressure \\ Research}

\begin{tabular}{l|l}
\hline Kidney Blood Press Res 2019;44:362-383 \\
\hline DOI: 10.1159/000501021 & $\begin{array}{l}\text { ○ 2019 The Author(s). Published by S. Karger AG, Basel } \\
\text { www.karger.com/kbr }\end{array}$ \\
\hline
\end{tabular}

Kataoka et al.: BMI-Based Cross-Classification Approach for CKD

\section{Definition of Subcohorts}

Four conventional attributes of CKD: age (age $\geq 65$ vs. $<65$ ), sex (male vs. female), CKD stage ( $4-5$ vs. 3 ), and (DM vs. non-DM) were used for cross classification. In this study, we initially examined the prognostic factors of CKD in the total cohort and subsequently divided the patients into 2 subcohorts by the BMI classification (BMI $<25 \mathrm{vs}$. BMI $\geq 25 \mathrm{~kg} / \mathrm{m}^{2}$ ). Finally, we made 4 subcohorts by multiplying the 4 conventional attributes by BMI classification, respectively. As patients were categorized into 4 mutually exclusive prespecified subcohorts on the basis of cross classification by BMI and conventional attributes, we were able to investigate the progression of CKD in a total of 16 subcohorts.

Outcomes

The primary outcome was a composite renal outcome, which was defined as a $\geq 50 \%$ decline in eGFR from baseline or ESRD, which requires renal replacement therapy. A $\geq 50 \%$ decline in eGFR from baseline was detected at the first of 3 consecutive visits when the renal event occurred. Renal replacement therapy was defined as any of hemodialysis, peritoneal dialysis, or kidney transplantation.

\section{Statistical Analysis}

Continuous variables are reported as means $\pm \mathrm{SD}$ or as medians (interquartile ranges); categorical variables are reported as percentages, unless otherwise stated. Group differences were evaluated using an analysis of variance, $\chi^{2}$-test, or Kruskal-Wallis test. To assess the primary endpoint, survival curves for 2 or 6 groups were plotted using the Kaplan-Meier method and evaluated using the log-rank test. Nonadjusted relationships between BMI and renal events were examined using Cox regression models with restricted cubic splines (knots were located at BMI values of 18.5, 23, 25, 27.5, and 30, and the reference point was a BMI of 22, in accordance with the WHO recommendations for Asian populations [11]). Univariate Cox regression analyses were performed; variables with a $p<0.1$ were selected for multivariate analyses. UACR was log-transformed. Multivariate Cox regression analyses using backward selection were performed $(p$ value for exclusion, $>0.10$ ) with multiple imputation using chained equations with predictive mean matching [12]. The number of imputations was 20 , and the imputation model included the variables marked with an asterisk $\left(^{*}\right)$ in Table 1, censoring indicators, and the Nelson-Aalen cumulative hazard estimator [13]. Interactions between BMI category and each predictor were considered by adding the BMI category and interaction term in the final model. Subgroup analyses were performed using the final model of the Cox regression analyses. Correlations between the identified prognostic predictors and other variables were assessed using the Pearson's correlation coefficient. Time-dependent receiver operating characteristics curves were estimated using the Kaplan-Meier estimator [14]. Calculated cutoff values minimized the distance from the point (sensitivity, 1 - specificity) $=(0,1)$ for the total cohort and all subcohorts. Sensitivity, specificity, positive likelihood ratio, negative likelihood ratio, diagnostic OR [15], and the area under the curve at 4 years were estimated, and a bootstrap method with 2,000 replications was used for $95 \% \mathrm{CI}$ estimation. Two-sided $p$ values $<0.05$ were considered statistically significant. All analyses were performed using SAS statistical software version 9.4 (SAS Institute Inc., Cary, NC, USA).

\section{Results}

\section{Patient Characteristics}

The characteristics of the 2,678 patients at baseline are shown in Table 1 . The subjects consisted of 1,685 males and 993 females, and their mean age at baseline was $60.4 \pm 11.5$ years. As shown in Table 1, the mean systolic blood pressure was $131.8 \pm 18.6 \mathrm{~mm} \mathrm{Hg}$, diastolic blood pressure $76.2 \pm 11.9 \mathrm{~mm} \mathrm{Hg}$, mean blood pressure was $94.7 \pm 12.8 \mathrm{~mm} \mathrm{Hg}$, PP $55.6 \pm$ $14.3 \mathrm{~mm} \mathrm{Hg}$, pulse pressure $\times$ heart rate product $(\mathrm{PP} \times \mathrm{HR}) 4,216.2 \pm 1,287.8 \mathrm{~mm} \mathrm{Hg} \times$ beats $/$ $\min$, BMI $23.5 \pm 3.8 \mathrm{~kg} / \mathrm{m}^{2}$, PWV 1,669.3 $\pm 472.1 \mathrm{~cm} / \mathrm{s}$, ankle-brachial index $1.11 \pm 0.13$, hemoglobin $12.1 \pm 1.9 \mathrm{~g} / \mathrm{dL}$, albumin $3.96 \pm 0.43 \mathrm{~g} / \mathrm{dL}$, uric acid $7.21 \pm 1.55 \mathrm{mg} / \mathrm{dL}$, potassium $4.64 \pm 0.58 \mathrm{mEq} / \mathrm{L}$, phosphorus $3.54 \pm 0.70 \mathrm{mg} / \mathrm{dL}$, LDL cholesterol $108.3 \pm 33.2 \mathrm{mg} / \mathrm{dL}$, HDL cholesterol $54.3 \pm 18.5 \mathrm{mg} / \mathrm{dL}$, estimated 24-h urinary potassium excretion (e24hUK) $38.6 \pm 8.0 \mathrm{mEq} /$ day, estimated 24 -h urinary sodium excretion $151.3 \pm 44.2 \mathrm{mEq} /$ day, and eGFR $28.8 \pm 12.3 \mathrm{~mL} / \mathrm{min} / 1.73 \mathrm{~m}^{2}$. The median UACR was $498.1(121.5-1,335.9) \mathrm{mg} / \mathrm{g}$ Cre 
Kidney

Blood Pressure

Research
Kidney Blood Press Res 2019;44:362-383

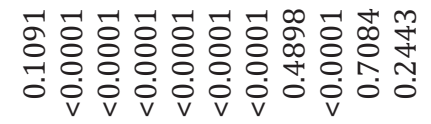

\begin{tabular}{l}
-1 \\
8 \\
8 \\
\hdashline
\end{tabular}

$\stackrel{\mathscr{N}}{\stackrel{\Xi}{\circ}}$

m $\exists \dot{G}$

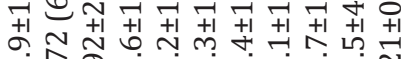

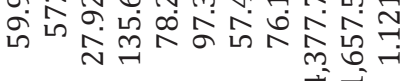

Fซล 仓્ల 능ํำ ฬอกล

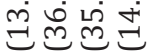

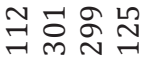
ํํํำ m F

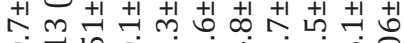

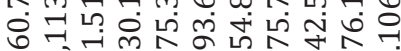

ลลกร

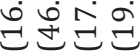
$\underbrace{\infty}_{\infty} \underbrace{}_{\infty}$

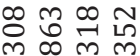

ละก స̃. 눈 늉 $\infty \infty \infty$ 6060 ciscis ลกำ

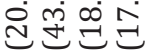

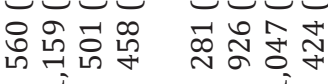

$\infty \infty \infty$ งิำ

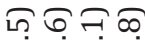
में - $\mathrm{i} \infty \mathrm{O}^{\circ}$.

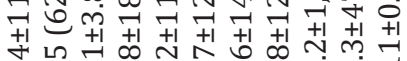

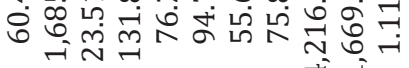
$6 \hat{0} \hat{0} \hat{0}$

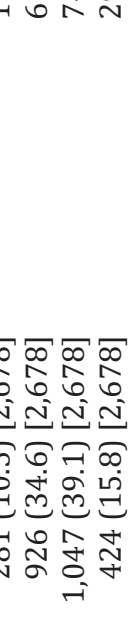

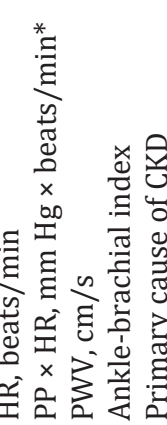

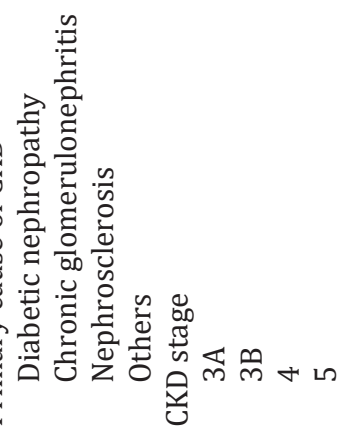

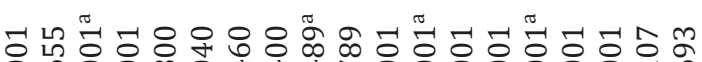

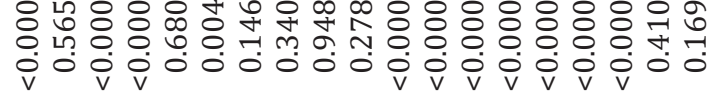

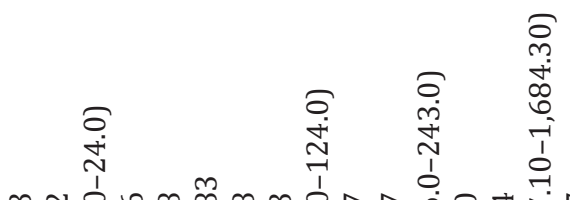
๓

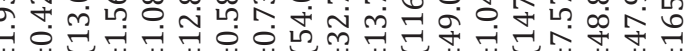
$+10$ 苻

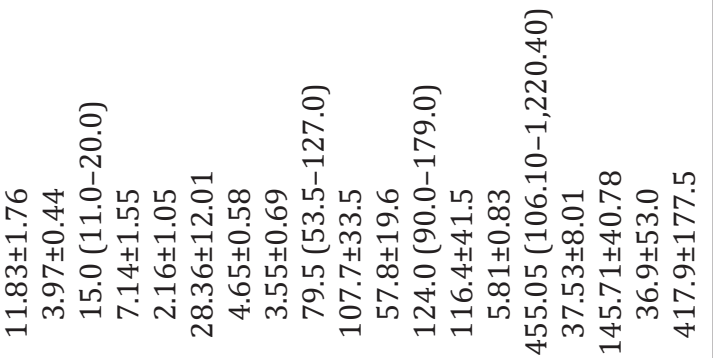

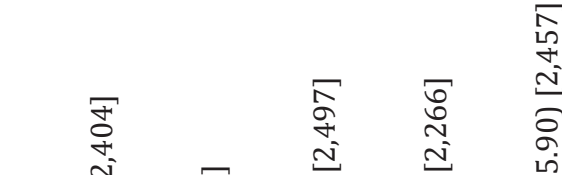
क

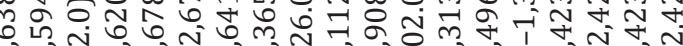
ง ñ

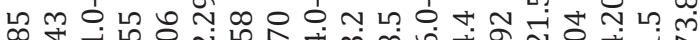
- o

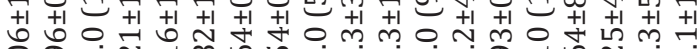
ำ $\rightarrow$ N

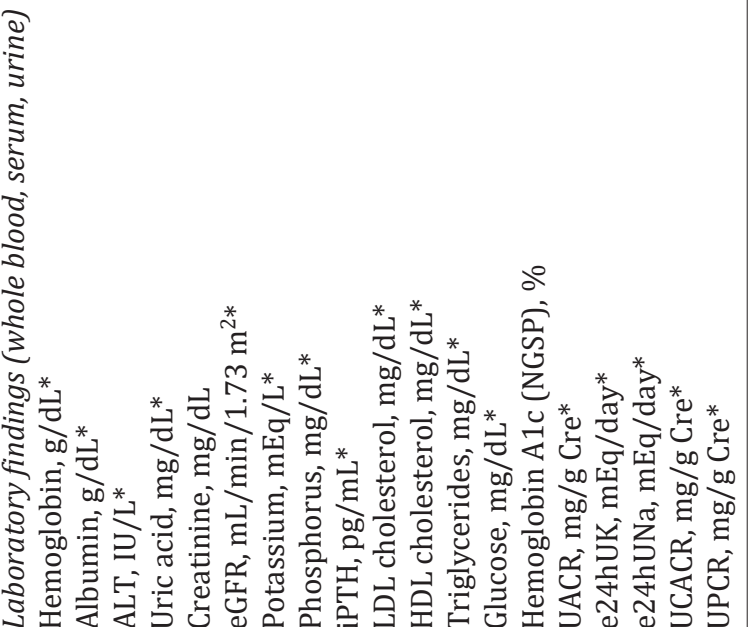


Research

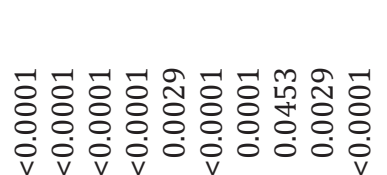

ㅈํำำำ 6 ำ

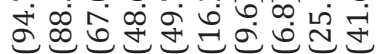

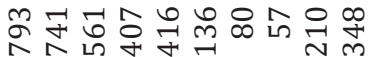

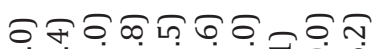
क्र

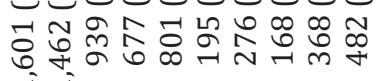

$\infty \infty \infty \infty \infty \infty \infty \infty$

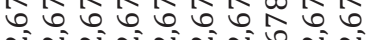

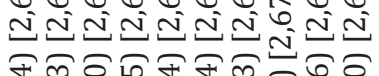
बin ळ

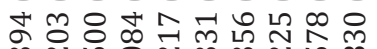

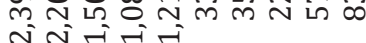

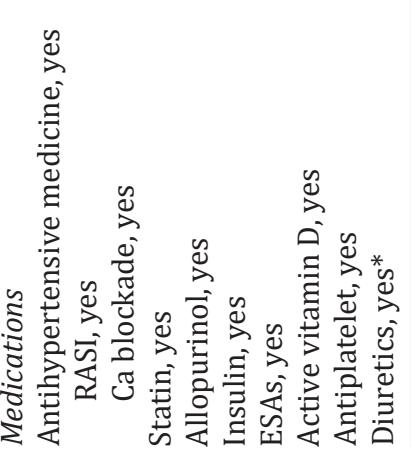

둥ㅎㅇㅇㅇㅇㅇㅇㅇㅇㅇ

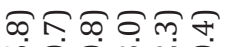
मूं

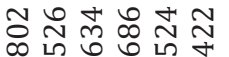

ส๘のสのร

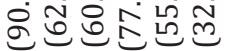
융ㅇㅇㅇㅢ

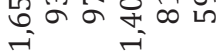

$\sigma \sigma \infty \pi \infty$ ถิำ

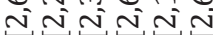
न片不。 สํู่ 웅 $m$ 눙

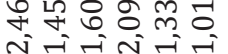

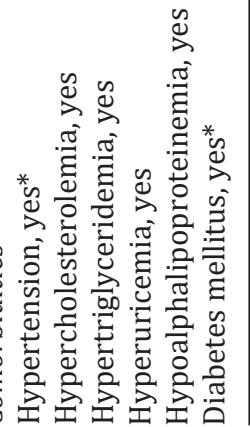

苛焉密

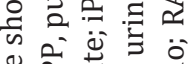

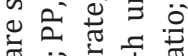

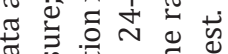
bo 艺进 元

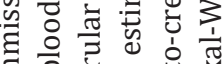
ป 웜 ส ডั

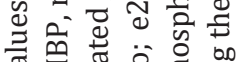

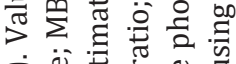
$\dot{*} \dot{0} \overline{0}$

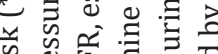

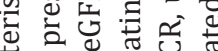

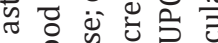
즈음 훙

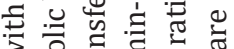

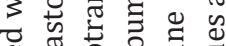
उั तै है 0 त 苟可 ن

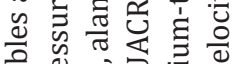
\%

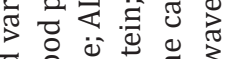

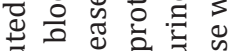
:气 :

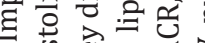

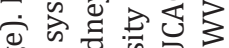

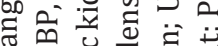
๑ ये 흘

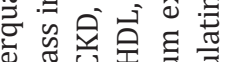
屯记元

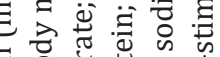

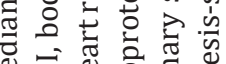

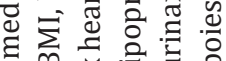
的 要焉站 +1 o o ब 0 व

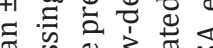
苋.

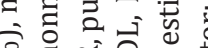

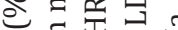
ธ。

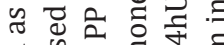
ర) के 중 $x \approx$ ¿ 인

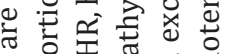

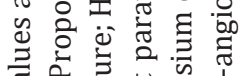

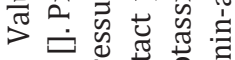




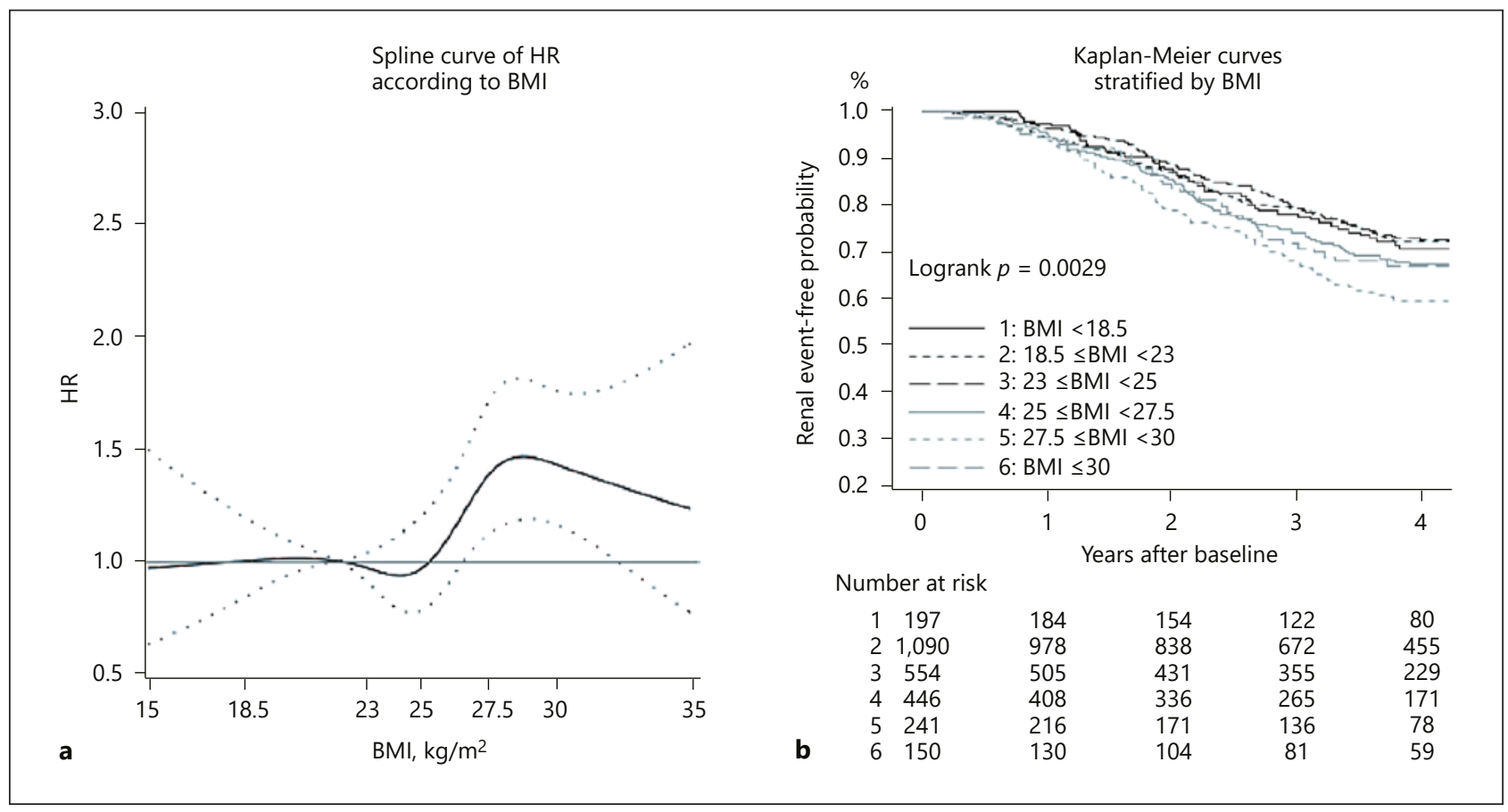

Fig. 1. Cubic spline curves according to BMI and Kaplan-Meier survival curves stratified by BMI (for 6 BMI categories in the total cohort). The cubic spline curves of the hazard ratio for renal outcomes according to BMI are shown for the total cohort (a). Model-based 95\% CIs are also reported (dashed lines). Kaplan-Meier curves stratified by BMI (6 BMI categories) are shown for the total cohort (b). HR, hazard ratio; BMI, body mass index.

and iPTH 79.0 (54.0-126.0) pg/mL. The medication data showed that 2,394 (89.4\%) were being treated with antihypertensive agents, 1,084 (40.5\%) with statin, 331 (12.4\%) with insulin, 830 (31.0\%) with diuretics, and $356(13.3 \%)$ with erythropoiesis-stimulating agents. The comorbidity data showed that 2,460 patients had hypertension, 1,456 had hypercholesterolemia, 1,604 had hypertriglyceridemia, and 1,018 had DM. During the follow-up period (median: 3.87 years, interquartile range: $2.04-4.22$ years), 811 patients reached the primary endpoint ( $\geq 50 \%$ eGFR decline or ESRD). The correlations of e24hUK with other clinical and laboratory parameters at baseline are shown in the online supplementary Figures (Appendix p 14: Appendix Fig. 5), and the characteristics at baseline and 4-year kidney survival of the subcohorts are shown in the online supplementary Tables (Appendix pp 41-64: Appendix Tables 1-4).

\section{Predictive Characteristics of the Attributes}

The predictive characteristics, including Kaplan-Meier survival curves and DORs, of the attributes used in the stratification and cross-classification analyses are illustrated in Figures 1-10 and the in the online supplementary Appendix (pp 7-12: Appendix Figs. 2, 3).

Increased BMI was associated with an increased risk of CKD progression in the range of BMI $\geq 25$ (Fig. 1a), which was observed in all subcohorts (Fig. 2b, c, 3a-d, 4a-c), with the exception of the subcohort of patients without DM (non-DM; Fig. 4d). Furthermore, in the elderly (age $\geq 65$ years) and female subcohorts, decreased BMI was also associated with an increased risk of CKD progression, resulting in U-shaped curves (Fig. 2c, Fig. 4b). KaplanMeier curves based on BMI stratification for 6 BMI categories indicated that patients with $27.5 \leq \mathrm{BMI}<30$ had the poorest prognosis (Fig. 1b: log-rank, $p=0.0029$ ). Kaplan-Meier curves 

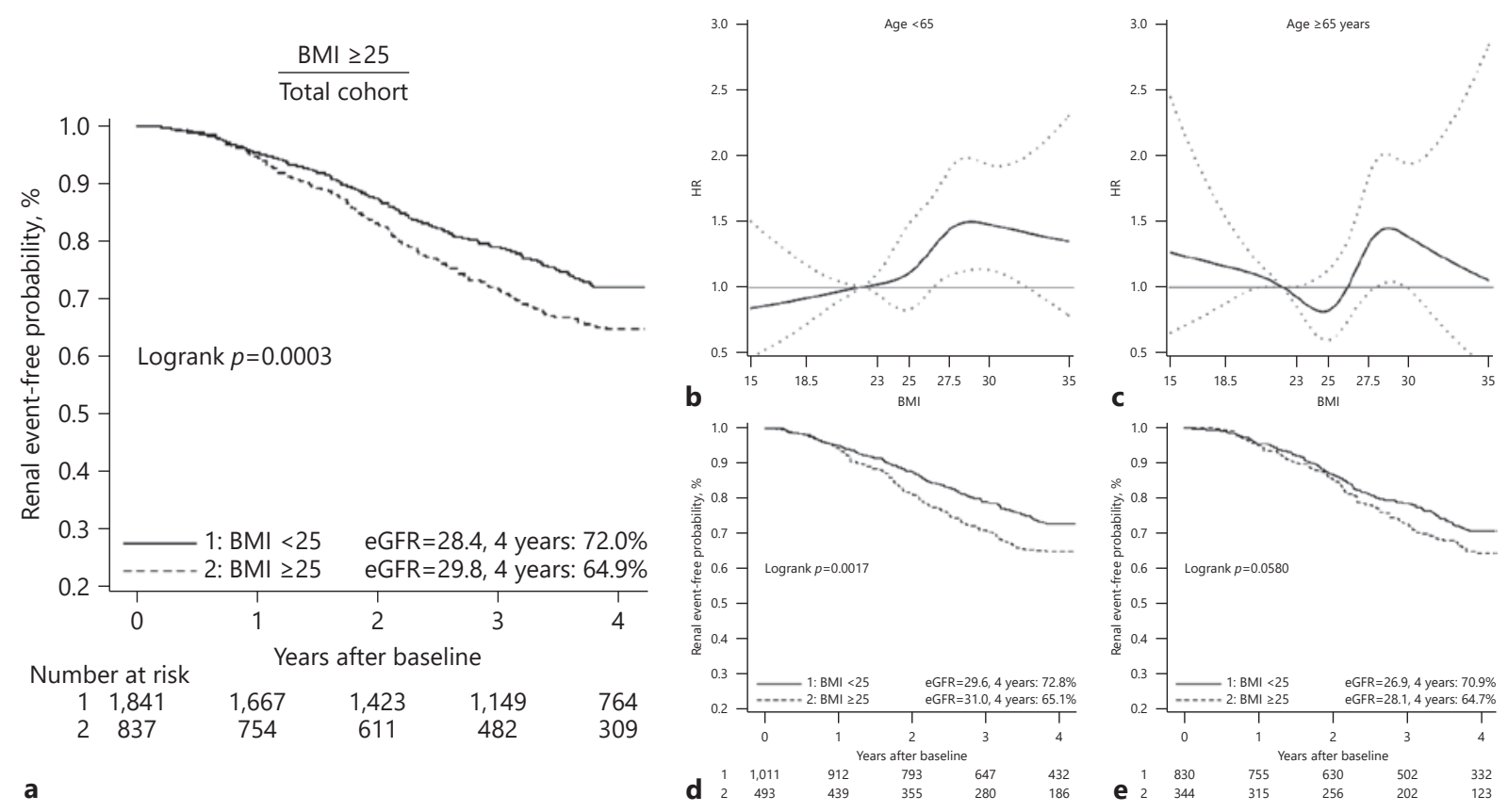

Fig. 2. Kaplan-Meier survival curves stratified by BMI (for 2 BMI categories in the total cohort) and age-based cubic spline curves according to BMI and Kaplan-Meier survival curves stratified by BMI. Kaplan-Meier survival curves stratified by BMI (2 BMI categories) are shown for the total cohort (a) and for age-based subcohorts (d, e). The cubic spline curves of the hazard ratio for renal outcomes according to BMI are shown for age-based subcohorts (b, c). Model-based 95\% CIs are also reported (dashed lines). BMI, body mass index; eGFR, estimated glomerular filtration rate; 4 years, 4-year survival rate; HR, hazard ratio.

based on BMI stratification for 2 BMI categories indicated that the renal survival rate of patients with $\mathrm{BMI} \geq 25$ was significantly lower than that for patients with $\mathrm{BMI}<25$ (Fig. 2a: log-rank, $p=0.0003$ ), which was observed in all subcohorts (Fig. 2d, e, 3e-h, 4e-g), with the exception of the elderly and non-DM subcohorts (Fig. 4h).

The sex-based Kaplan-Meier survival curves indicated that the renal survival rate was significantly lower in male patients than in female patients (Fig. 5a: log-rank, $p<0.0001$ ), which was observed in the nonelderly (age $<65$ years) and CKD stages $4-5$ subcohorts (Fig. $5 b$, $\mathrm{d}$; in the online suppl. Appendix p 8: Appendix Fig. 3a, right side of the panel A), but not in the elderly (age $\geq 65$ years), "BMI $\geq 25$, CKD stage 3" subcohorts (Fig. $5 c$, e; in the online suppl. Appendix p 8: Appendix Fig. 3a, left bottom of the panel A). The results for the DORs were similar (Fig. 6).

The age-based Kaplan-Meier survival curves for the total cohort did not demonstrate a significant difference between elderly and nonelderly subcohorts (Fig. 7a, log-rank, $p=0.8$ ). However, the renal survival rate of elderly patients was significantly lower than that for nonelderly patients in the "BMI $<25$, non-DM" and "BMI $<25$, female" subcohorts (log-rank; $p=0.002, p=0.03$, respectively; Fig. 7b; in the online suppl. Appendix p 9: Appendix Fig. 3b, right top of the panel $\mathrm{B}$ ). In contrast, the renal survival rate of elderly patients was significantly higher than that for nonelderly patients in the "BMI $<25$, DM" and "BMI $\geq 25, \mathrm{CKD}$ stages $4-5$ " subcohorts (log-rank; $p=0.0002, p<0.05$, respectively; Fig. $7 \mathrm{c}$; in the online suppl. Appendix p 9: Appendix Fig. 3B, right bottom of the panel A). The results for the DORs were similar (Fig. 8). 


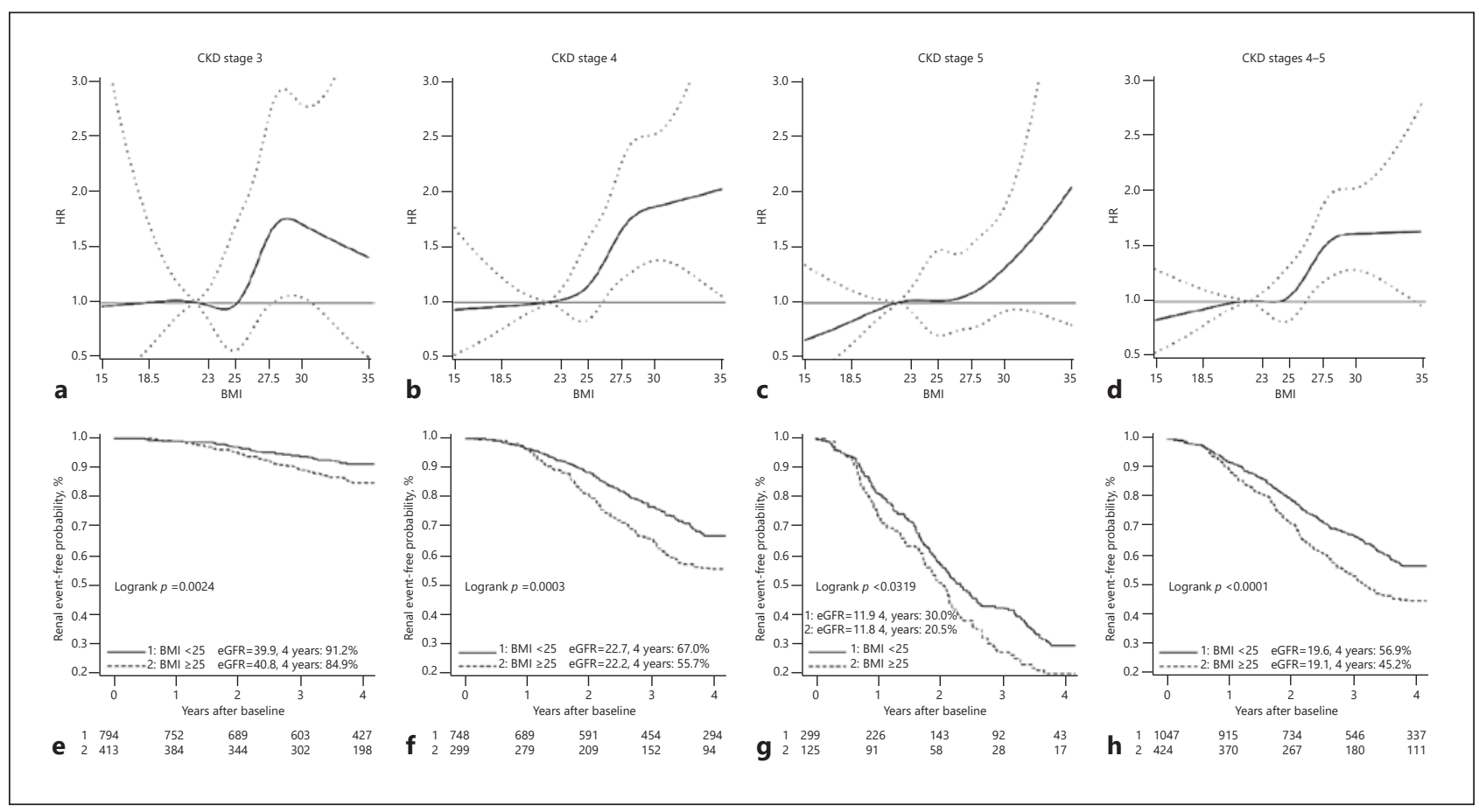

Fig. 3. CKD stage-based cubic spline curves according to BMI and Kaplan-Meier survival curves stratified by BMI. Cubic spline curves of the hazard ratio for renal outcomes according to BMI are shown for CKD stagebased subcohorts (a-d). Model-based 95\% CIs are also reported (dashed lines). Kaplan-Meier curves stratified by BMI are shown for CKD stage-based subcohorts (e-h). BMI, body mass index; eGFR, estimated glomerular filtration rate; 4 years, 4-year kidney survival rate; CKD, chronic kidney disease; HR, hazard ratio.

The CKD stage-based Kaplan-Meier survival curves indicated that the renal survival rate of patients with CKD stages 4-5 was significantly lower than that for patients with CKD stage 3 (in the online suppl. Appendix p 10: Appendix Fig. 3C-1, log-rank, $p<0.0001$ ), which was observed in all subcohorts (in the online suppl. Appendix pp 10, 11: Appendix Fig. 3C-1, 2). The results for the DORs were similar (in the online suppl. Appendix p 13: Appendix Fig. 4).

The DM-based Kaplan-Meier curves indicated that the renal survival rate of patients with DM was significantly lower than that of patients without DM (Fig. 9a, DM vs. non-DM, 60.7 vs. 75.5\%; log-rank, $p<0.0001$ ), which was observed in all subcohorts (Fig. 9b, d, e, in the online suppl. Appendix p 12: Appendix Fig. 3D), with the exception of the "BMI $<25$, age $\geq 65$ years" subcohort (Fig. 9c: DM vs. non-DM, 70.7 vs. $71.0 \%$; log-rank, $p=0.9359$ ). Similar results were obtained for the DORs (Fig. 10). The Appendix provides the results for elderly nonobese patients with DM (in the online suppl. Appendix pp 4, 62-64: supplementary results, Appendix Table 4).

\section{Primary Analyses}

Results of the univariate and multivariate regression analyses (Cox hazards models) for associations between the evaluated variables and primary endpoint in the total cohort are shown in Table 2.

The final model in the multivariate Cox analysis revealed the following risk factors as significantly associated with the primary endpoint: male sex; log UACR; increased phosphorus, PP $\times$ HR, BMI, and iPTH; and decreased e24hUK, hemoglobin, eGFR, age, and albumin (Table 2). Interactions between BMI category and each predictor on the risk of CKD progression were not significant, with the exception of eGFR and e24hUK. 


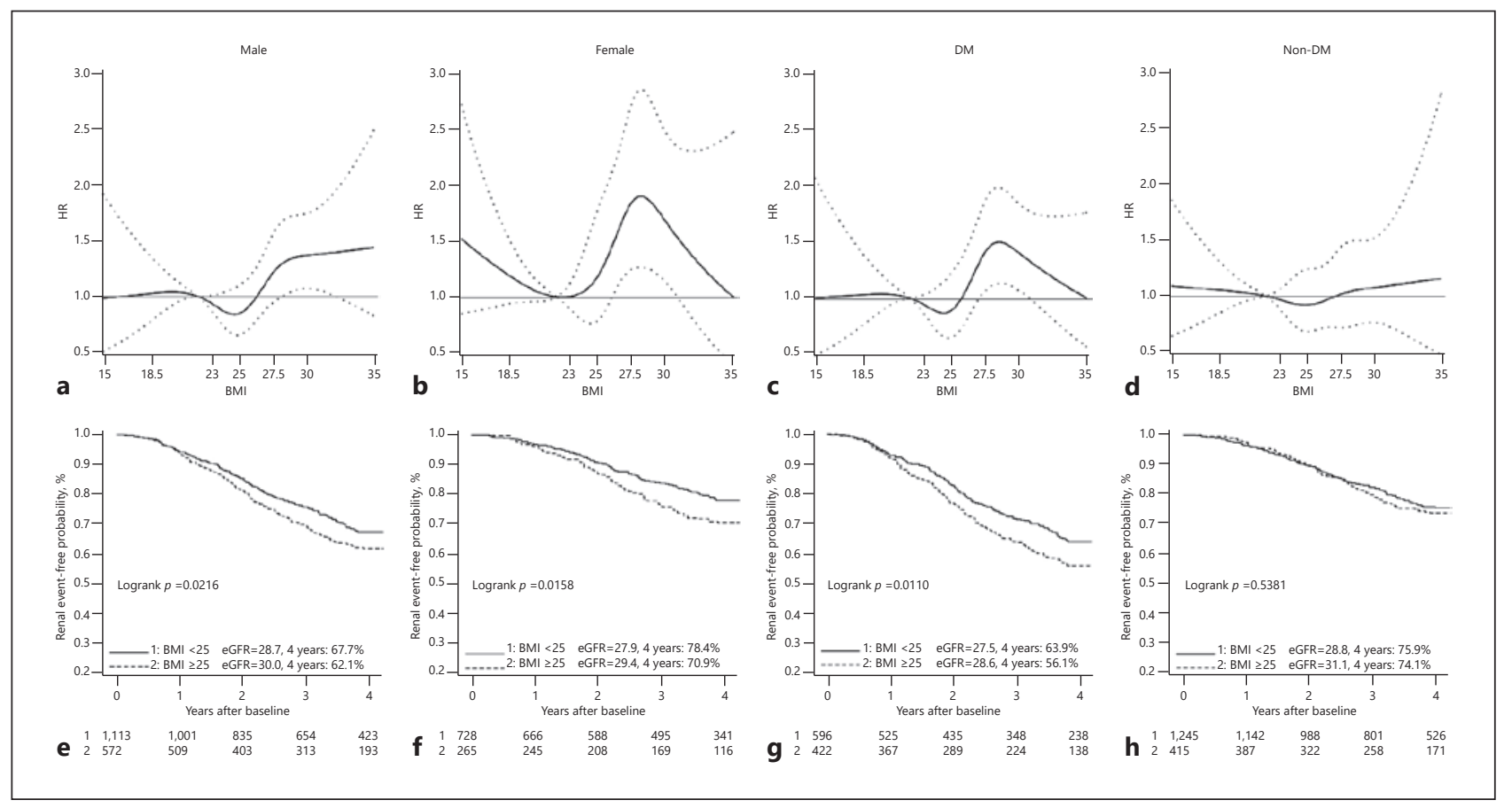

Fig. 4. Sex-based and DM-based cubic spline curves according to BMI and Kaplan-Meier survival curves stratified by BMI. Cubic spline curves of the hazard ratio for renal outcomes according to BMI are shown for sex-based (a, b) and DM-based subcohorts (c, d). Model-based 95\% CIs are also reported (dashed lines). Kaplan-Meier curves stratified by BMI are shown for sex-based (e, f) and DM-based subcohorts (g, h). BMI, body mass index; eGFR, estimated glomerular filtration rate; 4 years, 4-year kidney survival rate; DM, diabetes mellitus; HR, hazard ratio.

Prespecified subgroup Cox analyses were performed for all subcohorts (Fig. 11-15, in the online suppl. Appendix pp 68-71: Appendix Tables 6, 7). The number of prognostic factors identified in the multivariate Cox analysis was smaller in the "BMI $\geq 25$, female" (Fig. 12) and CKD stage 3 subcohorts (Fig. 14) than in other subcohorts. Prognostic factors identified in the "BMI $\geq 25$, CKD stage 3" subcohort only comprised log UACR and male sex (Fig. 14), and those in the "BMI $\geq 25$, female" subcohort only comprised log UACR, phosphorus, and hemoglobin (Fig. 12).

As shown in Figures 11-15, log UACR, eGFR, male sex, phosphorus, hemoglobin, and $\mathrm{PP} \times \mathrm{HR}$ were stable renal prognostic factors in almost all subcohorts. On the other hand, the prognostic value of BMI, age, serum albumin, iPTH, and e24hUK differed according to subcohort. PP $\times$ HR, which reflects pulsatile stress, was independently associated with CKD progression in most of subcohorts, including the "BMI <25, CKD stage 3" subcohort (Fig. 14). Reduced urinary potassium excretion was a limited renal prognostic factor for CKD progression in the "BMI $<25$, CKD stages $4-5$ " subcohort, but not for the "BMI $\geq 25$, CKD stages 4-5" subcohort (Fig. 14). BMI was positively associated with CKD progression in the "BMI $\geq 25$, age $\geq 65$ years" and "BMI $\geq 25$ (Fig. 13), CKD stages $4-5$ " subcohorts (Fig. 14), whereas it was negatively associated with CKD progression in the "BMI $<25$, DM" subcohort (Fig. 15).

\section{Characteristics of $P P \times H R$}

As $\mathrm{PP} \times \mathrm{HR}$ was a newly identified prognostic predictor of CKD progression, the correlations of PP $\times$ HR with other clinical and laboratory parameters at baseline were examined. As 

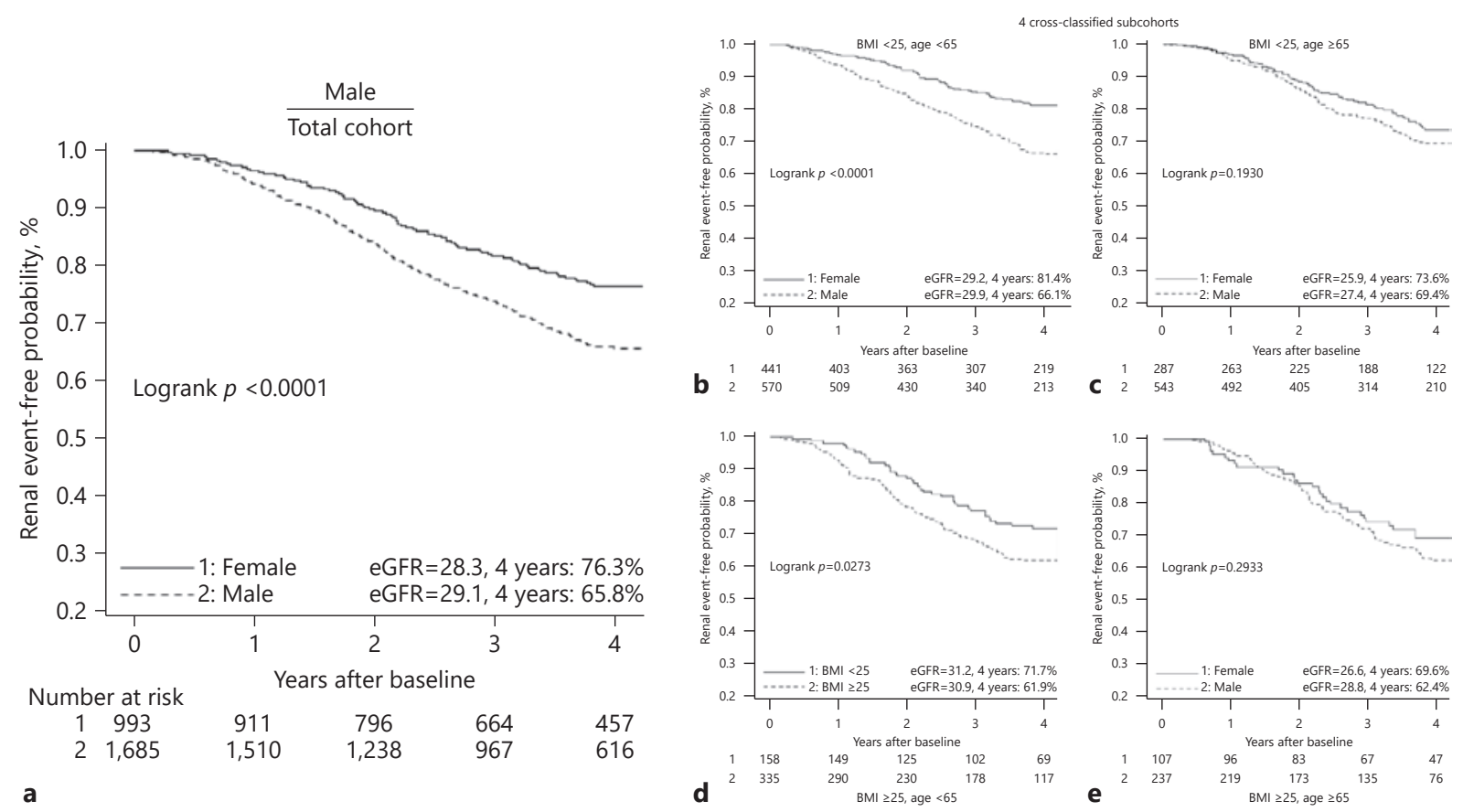

Fig. 5. Kaplan-Meier survival curves stratified by sex. Kaplan-Meier survival curves stratified by sex are shown for the total cohort (a) and 4 BMI-based cross-classified subcohorts (b-e). BMI, body mass index; eGFR, estimated glomerular filtration rate; 4 years, 4-year kidney survival rate.

shown in Figure 16, PP $\times$ HR was positively correlated with age $(r=0.22, p<0.0001)$, UACR $(r=0.27, p<0.0001)$, and PWV $(r=0.52, p<0.0001) . \mathrm{PP} \times \mathrm{HR}$ was more positively correlated with age in females $(r=0.31)$ than in males $(r=0.16)$ in a stratified analysis with sex.

\section{Supplementary Analyses}

With consideration for accessibility in clinical settings, the prognostic predictors identified in the final Cox regression analyses model were dichotomized using cutoff values determined by time-receiver operating characteristics curves. The prognostic effects of CKD progression were confirmed using the DOR and Kaplan-Meier method for all cohorts (in the online suppl. Appendix pp 15-36, 72-76: Appendix Fig. 6-8, Appendix Table 8; and Appendix pp 15-16, 37-40: Appendix Fig. 9).

\section{Discussion}

Recently, patient-centered medicine (PCM) has been advocated [16, 17]. For PCM, it is necessary to treat individual patients according to their heterogeneous characteristics [18]. The development of PCM requires patient-oriented research, focused on the individualization of results, disaggregation of data, and analysis of differences in subgroups [19]. Patients generally have many attributes, thus, previous studies using conventional subgroup analyses are limited, as classifications were based on a single variable (e.g., male vs. female, elderly vs. young) [16]. One-variable-at-a-time comparisons usually result in the subcohorts being more similar to the total cohort because patients simultaneously have multiple 
Fig. 6. DORs of males according to subcohorts. A summary of online supplementary Appendix Table 5 is depicted. DORs of males for a decline in estimated glomerular filtration rate by $\geq 50 \%$ from baseline or ESRD during the follow-up examination period are shown according to subcohort. BMI, body mass index; CKD, chronic kidney disease; DM, diabetes mellitus; DOR, diagnostic odd ratios.

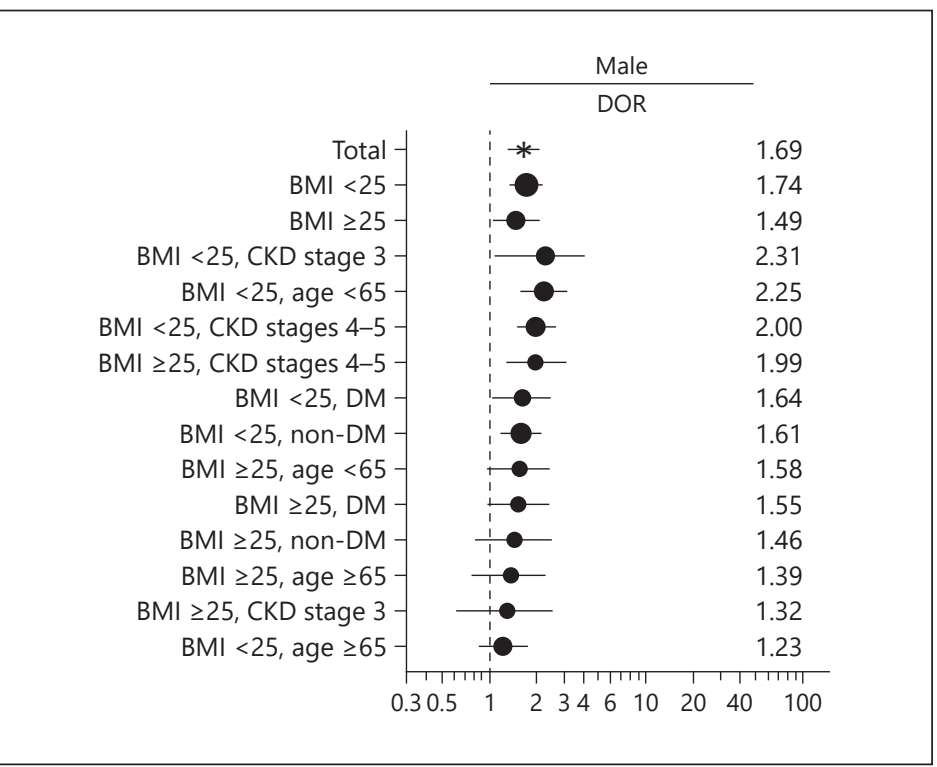

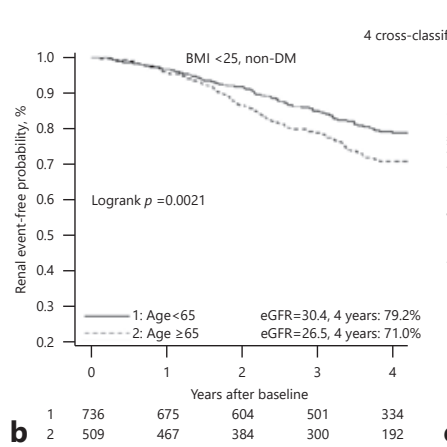
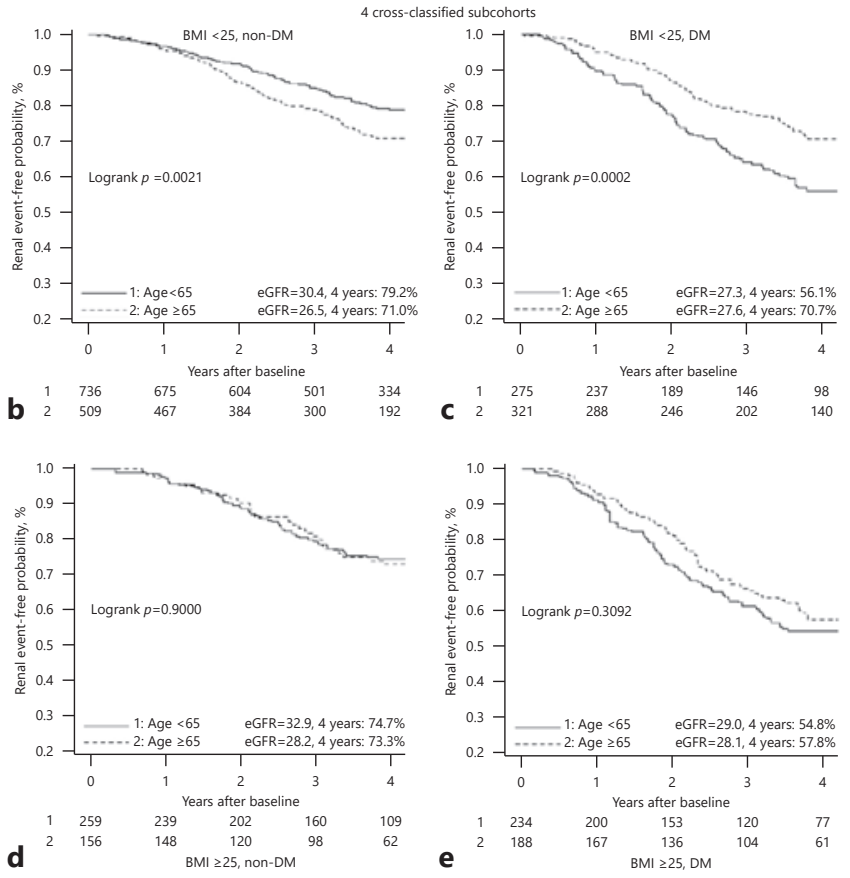

Fig. 7. Kaplan-Meier survival curves stratified by age. Kaplan-Meier survival curves stratified by age are shown for the total cohort (a) and 4 BMI-based cross-classified subcohorts (b-e). BMI, body mass index; eGFR, estimated glomerular filtration rate; 4 years, 4-year survival rate; DM, diabetes mellitus.

characteristics [20], which was confirmed in our analyses as illustrated in the in the online supplementary Appendix (pp 7, 17-18: Appendix Fig. 2, 7). To overcome this limitation, we used cross classification, enabling effective classification using 2 attributes simultaneously. Subgroup analysis by cross classification allows the determination of positive and negative 
Fig. 8. DORs of elderly patients according to subcohort. A summary of online supplementary Appendix Table 5 is depicted. DORs of elderly patients (age $\geq 65$ years) for a decline in eGFR by $\geq 50 \%$ from baseline or ESRD during the follow-up examination period are shown according to subcohort. BMI, body mass index; CKD, chronic kidney disease; DM, diabetes mellitus; DOR, diagnostic odd ratios.
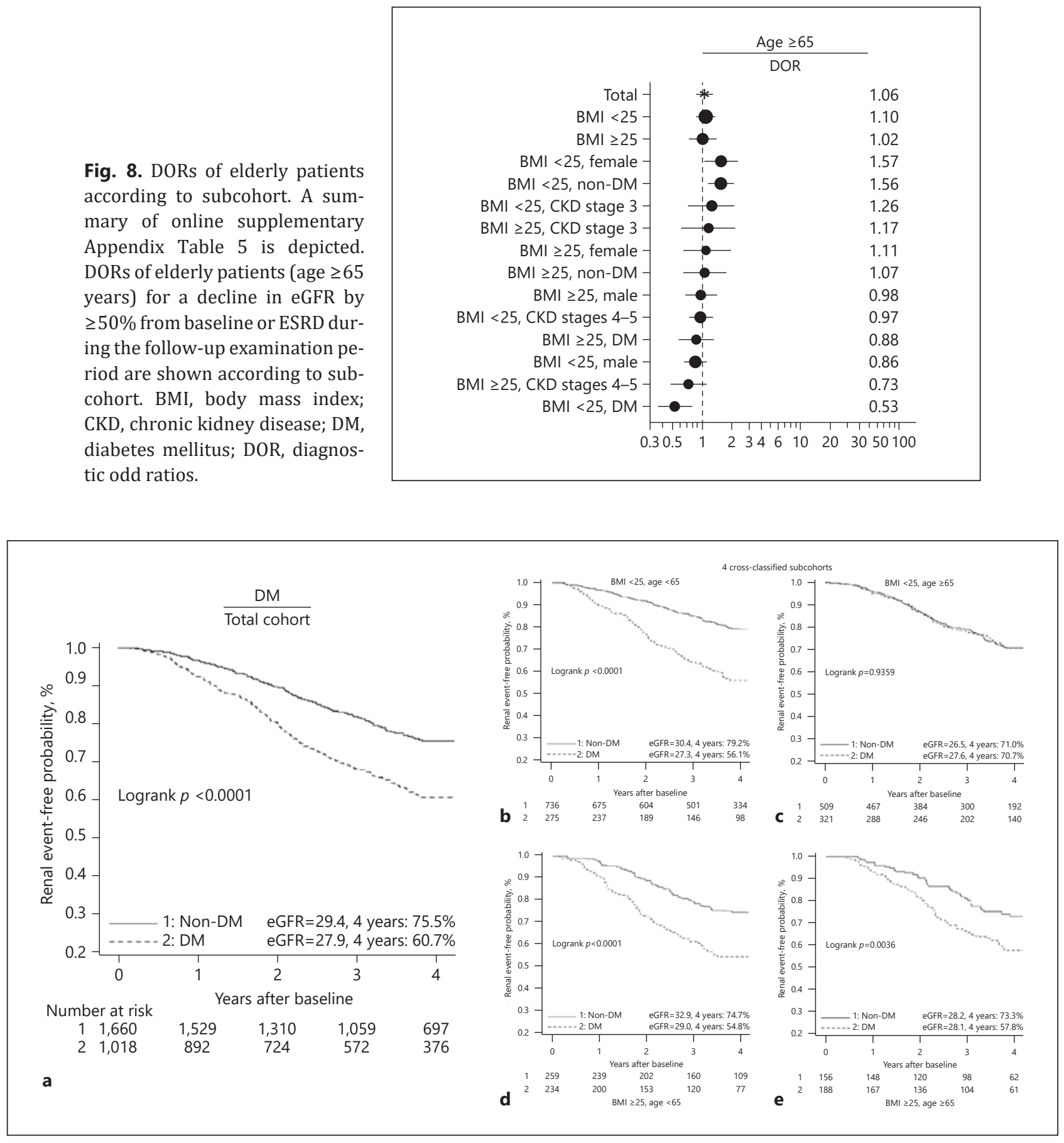

Fig. 9. Kaplan-Meier survival curves stratified by DM status. Kaplan-Meier survival curves stratified by DM status are shown for the total cohort (a) and 4 BMI-based cross-classified subcohorts (b-e). BMI, body mass index; eGFR, estimated glomerular filtration rate; 4 years, 4-year survival rate; DM, diabetes mellitus.

findings that identify which subcohort(s) will and will not benefit from a treatment intervention, respectively. In the future, research utilizing large datasets may become more popular, for which, cross-classification analysis will be highly useful. In the present study, the positive findings were that PP $\times$ HR is a prognostic factor in patients in the "BMI $<25$, 
Kataoka et al.: BMI-Based Cross-Classification Approach for CKD

Fig. 10. DORs of DM according to subcohort. A summary of online supplementary Appendix Table 5 is depicted. DORs of DM for a decline in eGFR by $\geq 50 \%$ from baseline or ESRD during the follow-up examination period are shown according to subcohort. BMI, body mass index; CKD, chronic kidney disease; DM, diabetes mellitus; DOR, diagnostic odd ratios.

Fig. 11. Multivariate Cox analysis for prognostic predictors in subcohorts stratified by BMI. A summary of online supplementary Appendix Table 6 is depicted. The results of the multivariate Cox analysis for the prognostic predictors associated with a $\geq 50 \%$ eGFR decline or ESRD in subcohorts stratified by BMI are shown. BMI, body mass index; eGFR, estimated glomerular filtration rate; UACR, urine albumin-to-creatinine ratio; e24hUK, estimated 24-h urinary potassium excretion; $\mathrm{PP} \times \mathrm{HR}$, pulse pressure $\times$ heart rate product; iPTH, intact parathyroid hormone; HR, hazard ratio.

\section{2 stratified subcohorts}

BMI <25: $n=1,841$, eGFR $28.4 \mathrm{~mL} / \mathrm{min} / 1.73 \mathrm{~m}^{2}$, 4-year survival rate $72.0 \%$

- Male HR 2.63, $p<0.0001$

- $\log$ UACR $(/ 1 \mathrm{log}(\mathrm{mg} / \mathrm{g}$ Cre $)) \quad$ HR 1.83, $p<0.0001$

- e24hUK (/10 mEq/day)

- Phosphorus (/1 mg/dL)

- Hemoglobin $(/ 1 \mathrm{~g} / \mathrm{dL})$

HR $0.70, p<0.0001$

HR 1.41, $p<0.0001$

HR $0.83, p<0.0001$

HR $0.93, p<0.0001$

- eGFR (/1 mL/min/1.73 m²)

- PP $\times$ HR (/1,000 mm Hg-bpm)

- Age (/1 year)

HR 1.16, $p=0.0005$

HR 0.99, $p=0.0337$

- iPTH $(/ 10 \mathrm{pg} / \mathrm{mL})$ HR 1.01, $p=0.0338$

$\mathrm{BMI} \geq 25: n=837$, eGFR $29.8 \mathrm{~mL} / \mathrm{min} / 1.73 \mathrm{~m}^{2}$, 4-year survival rate $64.9 \%$

- Male

- $\log$ UACR (/1 log(mg/g Cre))

- eGFR (/1 mL/min/1.73 m²)

- Phosphorus (/1 mg/dL)

- Hemoglobin (/1 g/dL)

- $\mathrm{PP} \times \mathrm{HR}(/ 1,000 \mathrm{~mm} \mathrm{Hg}-\mathrm{bpm})$

- Age (/1 year)

HR 1.14, $p=0.0241$

HR 0.99, $p=0.0328$

CKD stage 3" subcohort, and that e24hUK has importance for renal outcomes in patients in the "BMI <25, CKD stages 4-5" subcohort in the multivariate Cox analyses, whereas the negative finding was that DM did not affect the renal prognosis in patients in the "BMI $<25$, age $\geq 65$ years" subcohort in the Kaplan-Meier analyses. Furthermore, as shown in the online supplementary results in the Appendix, we found differences in the cutoff values for renal anemia and hyperphosphatemia according to subcohort in the cross-classification analyses.

Conventional attributes, such as age, sex, CKD stage, and DM, have been reported as prognostic factors for renal outcomes. Many studies suggest that male sex is associated with worse renal outcomes [21-24], consistent with the present study (Table 2). Sex differences exist in the pathogenesis and clinical prognosis of CKD [25], and female sex seems to be renoprotective for the development and progression of CKD. Interestingly, in the Kaplan- 


\begin{tabular}{|c|c|c|c|}
\hline \multicolumn{4}{|c|}{4 cross-classified subcohorts } \\
\hline \multicolumn{2}{|c|}{$\begin{array}{ll}\text { BMI }<25, \text { male: } n=1,113 \text {, eGFR 28.7, } 4 \text {-year } 67.7 \% \\
\text { - log UACR }(/ 1 \mathrm{log}(\mathrm{mg} / \mathrm{g} \text { Cre })) & \text { HR } 1.88, p<0.0001 \\
\text { - e24hUK }(/ 10 \mathrm{mEq} / \mathrm{day}) & \text { HR } 0.66, p<0.0001 \\
\text { - Phosphorus }(/ 1 \mathrm{mg} / \mathrm{dL}) & \text { HR } 1.44, p<0.0001 \\
\text { - Hemoglobin }(/ 1 \mathrm{~g} / \mathrm{dL}) & \text { HR } 0.84, p<0.0001 \\
\text { - eGFR }\left(/ 1 \mathrm{~mL} / \mathrm{min} / 1.73 \mathrm{~m}^{2}\right) & \text { HR } 0.94, p<0.0001 \\
\text { - iPTH }(/ 10 \mathrm{pg} / \mathrm{mL}) & \text { HR } 1.02, p=0.0089 \\
\text { - PP } \times \text { HR }(/ 1,000 \mathrm{~mm} \mathrm{Hg} \text {-bpm) } & \text { HR } 1.12, p=0.0389 \\
\text { - Age }(/ 1 \text { year) } & \text { HR } 0.99, p=0.0489\end{array}$} & \multicolumn{2}{|c|}{ 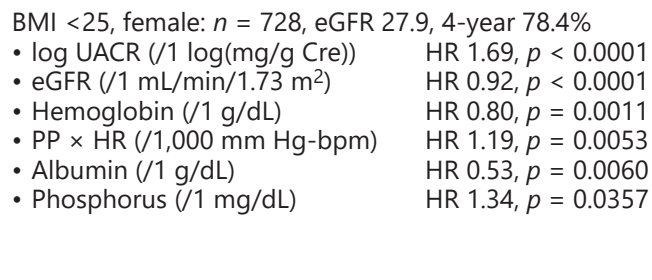 } \\
\hline $\begin{array}{l}\text { BMI } \geq 25 \text {, male: } n=572, \text { eGFR } 30 \text {. } \\
\text { - log UACR }(/ 1 \mathrm{log}(\mathrm{mg} / \mathrm{g} \text { Cre })) \\
\text { - eGFR }\left(/ 1 \mathrm{~mL} / \mathrm{min} / 1.73 \mathrm{~m}^{2}\right) \\
\text { - Albumin }(/ 1 \mathrm{~g} / \mathrm{dL}) \\
\text { - Hemoglobin }(/ 1 \mathrm{~g} / \mathrm{dL}) \\
\text { - Phosphorus }(/ 1 \mathrm{mg} / \mathrm{dL}) \\
\text { - iPTH }(/ 10 \mathrm{pg} / \mathrm{mL}) \\
\text { - Age }(/ 1 \text { year) } \\
\text { - PP } \times \text { HR }(/ 1,000 \mathrm{~mm} \mathrm{Hg} \text {-bpm) }\end{array}$ & $\begin{array}{l}\text { 4-year } 62.1 \% \\
\text { HR } 1.79, p<0.0001 \\
\text { HR } 0.95, p<0.0001 \\
\text { HR } 0.62, p=0.0060 \\
\text { HR } 0.85, p=0.0055 \\
\text { HR } 1.30, p=0.0135 \\
\text { HR } 1.02, p=0.0089 \\
\text { HR } 0.98, p=0.0408 \\
\text { HR } 1.14, p=0.0446\end{array}$ & $\begin{array}{l}\text { BMI } \geq 25, \text { female: } n=265 \text {, eGFR } 29 \\
\text { - log UACR (/1 log(mg/g Cre }) \text { ) } \\
\text { - Phosphorus (/1 mg/dL) } \\
\text { - Hemoglobin }(/ 1 \mathrm{~g} / \mathrm{dL})\end{array}$ & $\begin{array}{l}.4,4 \text {-year } 70.9 \% \\
\text { HR 2.37, } p<0.0001 \\
\text { HR 1.89, } p=0.0325 \\
\text { HR 0.81, } p=0.0499\end{array}$ \\
\hline
\end{tabular}

Fig. 12. Multivariate Cox analysis for prognostic predictors in subcohorts cross classified by sex and BMI. A summary of online supplementary Appendix Table 7A is depicted. The results of the multivariate Cox analysis for the prognostic predictors associated with a $\geq 50 \%$ eGFR decline or ESRD in subcohorts cross classified by sex and BMI are shown. BMI, body mass index; eGFR, estimated glomerular filtration rate; 4-year, 4-year survival rate; UACR, urine albumin-to-creatinine ratio; e24hUK, estimated 24-h urinary potassium excretion; iPTH, intact parathyroid hormone; PP $\times$ HR, pulse pressure $\times$ heart rate product; HR, hazard ratio.

\begin{tabular}{|c|c|c|c|}
\hline \multicolumn{4}{|c|}{4 cross-classified subcohorts } \\
\hline \multicolumn{2}{|c|}{$\begin{array}{ll}\text { BMI }<25 \text {, age }<65: n=1,011 \text {, eGFR } 29.6,4 \text {-year } 72.8 \% \\
\text { - Male } & \text { HR 3.13, } p<0.0001 \\
\text { - log UACR }(/ 1 \mathrm{log}(\mathrm{mg} / \mathrm{g} \text { Cre })) & \text { HR } 1.84, p<0.0001 \\
\text { - Hemoglobin }(/ 1 \mathrm{~g} / \mathrm{dL}) & \text { HR } 0.83, p<0.0001 \\
\text { - eGFR }\left(/ 1 \mathrm{~mL} / \mathrm{min} / 1.73 \mathrm{~m}^{2}\right) & \text { HR } 0.92, p<0.0001 \\
\text { - Phosphorus }(/ 1 \mathrm{mg} / \mathrm{dL}) & \text { HR } 1.37, p=0.0017 \\
\text { - PP } \times \text { HR }(/ 1,000 \mathrm{~mm} \mathrm{Hg} \text {-bpm) } & \text { HR } 1.15, p=0.0230 \\
\text { - e24hUK }(/ 10 \mathrm{mEq} / \mathrm{day}) & \text { HR } 0.79, p=0.0391 \\
\text { - Albumin }(/ 1 \mathrm{~g} / \mathrm{dL}) & \text { HR } 0.74, p=0.0464\end{array}$} & \multicolumn{2}{|c|}{ 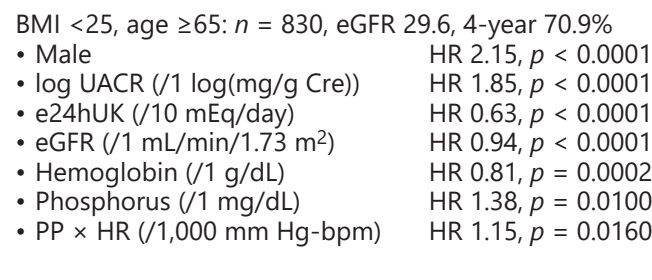 } \\
\hline $\begin{array}{l}\text { BMI } \geq 25 \text {, age }<65: n=493, \text { eGFR } \\
\text { - Male } \\
\text { - log UACR }(/ 1 \mathrm{log}(\mathrm{mg} / \mathrm{g} \text { Cre })) \\
\text { - eGFR }\left(/ 1 \mathrm{~mL} / \mathrm{min} / 1.73 \mathrm{~m}^{2}\right) \\
\text { - Phosphorus }(/ 1 \mathrm{mg} / \mathrm{dL}) \\
\text { - Age }(/ 1 \text { year }) \\
\text { - Albumin }(/ 1 \mathrm{~g} / \mathrm{dL})\end{array}$ & $\begin{array}{l}31.0,4 \text {-year } 65.1 \% \\
\text { HR 2.59, } p<0.0001 \\
\text { HR 2.10,p }<0.0001 \\
\text { HR 0.95, } p<0.0001 \\
\text { HR } 1.49, p=0.0002 \\
\text { HR } 0.97, p=0.0055 \\
\text { HR } 0.67, p=0.0457\end{array}$ & $\begin{array}{l}\text { BMI } \geq 25, \text { age } \geq 65: n=344 \text {, eGFR } \\
\text { - Male } \\
\text { - log UACR }(/ 1 \mathrm{log}(\mathrm{mg} / \mathrm{g} \text { Cre })) \\
\text { - Hemoglobin }(/ 1 \mathrm{~g} / \mathrm{dL}) \\
\text { - eGFR }\left(/ 1 \mathrm{~mL} / \mathrm{min} / 1.73 \mathrm{~m}^{2}\right) \\
\text { - BMI }\left(/ 1 \mathrm{~kg} / \mathrm{m}^{2}\right) \\
\text { - PP } \times \mathrm{HR}(/ 1,000 \mathrm{~mm} \mathrm{Hg}-\mathrm{bpm})\end{array}$ & $\begin{array}{rl}28.1,4-y e a r & 64.7 \% \\
\text { HR 2.97, } p<0.0001 \\
\text { HR } 1.92, p<0.0001 \\
\text { HR } 0.68, p<0.0001 \\
\text { HR } 0.96, p=0.0024 \\
\text { HR } 1.15, p=0.0111 \\
\text { HR } 1.23, p=0.0240\end{array}$ \\
\hline
\end{tabular}

Fig. 13. Multivariate Cox analysis for prognostic predictors in subcohorts cross classified by age and BMI. A summary of online supplementary Appendix Table 7B is depicted. The results of the multivariate Cox analysis for the prognostic predictors associated with a $\geq 50 \%$ eGFR decline or ESRD in subcohorts cross classified by age and BMI are shown. BMI, body mass index; eGFR, estimated glomerular filtration rate; 4-year, 4-year survival rate; UACR, urine albumin-to-creatinine ratio; $\mathrm{PP} \times \mathrm{HR}$, pulse pressure $\times$ heart rate product; $\mathrm{e} 24 \mathrm{hUK}$, estimated 24-h urinary potassium excretion; HR, hazard ratio.

Meier analysis, this female renal survival advantage disappeared in the elderly subcohort (Fig. 5c, e), suggesting that this advantage is neutralized with aging or menopause. Although sex is a renal prognostic factor, considering its unmodifiable characteristics, it is reasonable to use it as an attribute. 


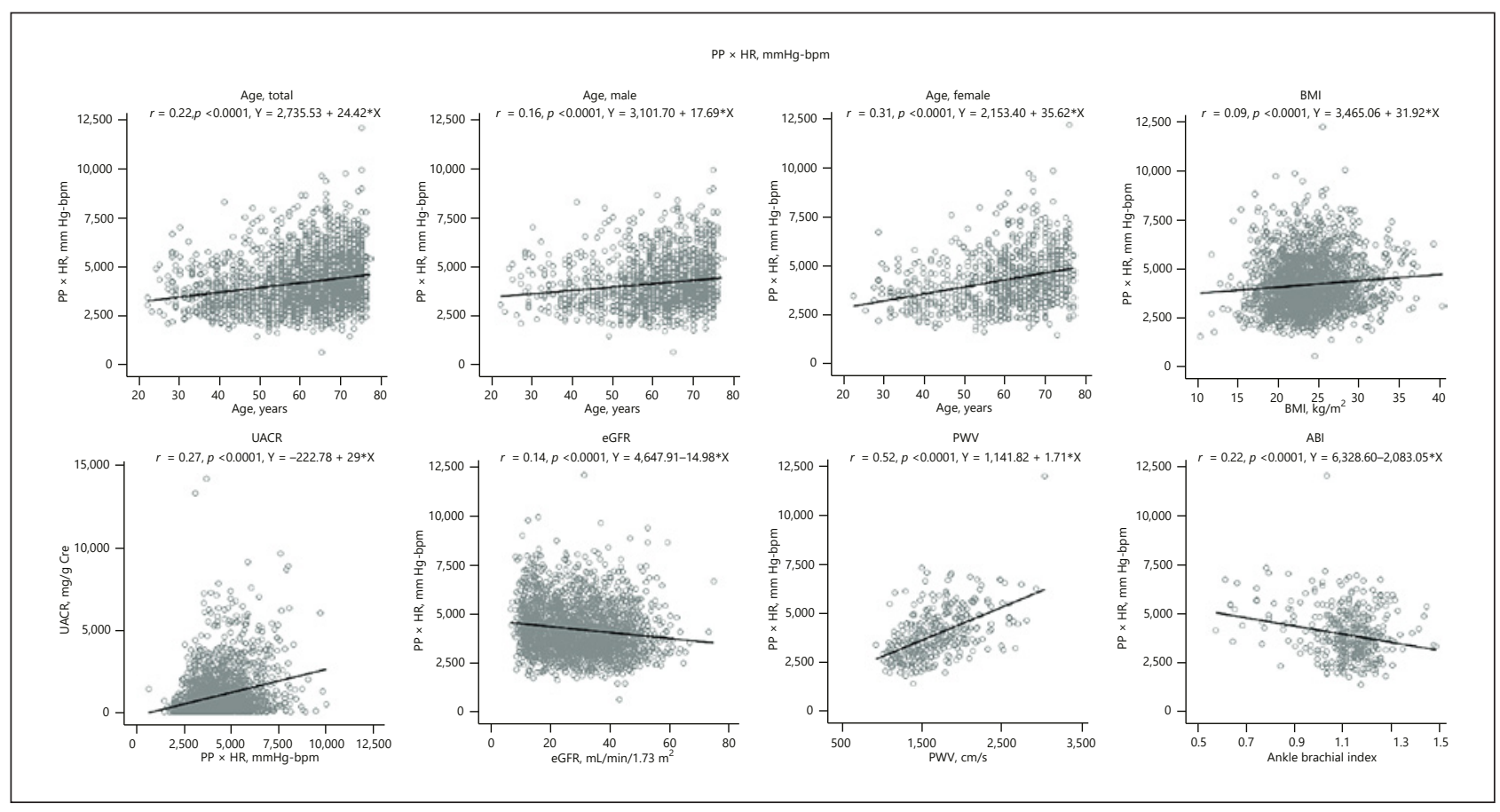

Fig. 16. Relationship between the double product of $\mathrm{PP} \times \mathrm{HR}$ with baseline variables. The analyses in relation to age were stratified by sex. BMI, body mass index; UACR, urine albumin-to-creatinine ratio; eGFR, estimated glomerular filtration rate; PWV, pulse wave velocity; ABI, ankle-brachial index; $\mathrm{PP} \times \mathrm{HR}$, pulse pressure $\times$ heart rate product.

The results of studies on elderly status as a prognostic factor for renal prognosis have been inconsistent [21]. In the present study, although elderly status was associated with slightly better renal outcomes in the total cohort (Table 2), the prognostic characteristics of elderly patients in the Kaplan-Meier analyses differed according to the BMI-based cross-classified subcohort (Fig. 7b-e). Considering that there may be pathophysiological differences between elderly and younger adult patients with CKD [26,27], it is reasonable to use age as an attribute in clinical studies.

Consistent with the present study results (Table 2), many studies suggest that decreased GFR is associated with worse renal outcomes [28]. Considering that reductions in GFR are associated with CKD complications that may lead to CKD progression, a subanalysis according to CKD stage is important and it is reasonable to use CKD stage as an attribute.

DM is generally considered a prognostic factor for the development and progression of CKD [21]. Although the total-cohort multivariate Cox analysis did not show significant differences in renal prognostic factors between patients with and without DM, the Kaplan-Meier analysis for the total cohort showed a significantly worse prognosis in terms of the 4-year renal survival rate in the patients with DM (Fig. 9a). On the other hand, the Kaplan-Meier curves for the "BMI $<25$, age $\geq 65$ years" subcohort in both the DM and non-DM groups almost overlapped (Fig. 9c). Although further investigation is needed, there is a possibility that DM does not affect the renal prognosis in nonoverweight elderly patients. Despite the high diabetes prevalence in the elderly population, evidence of treatment effects is deficient, especially regarding the lowering of glucose levels [29]. Our BMI-based cross-classification analysis indicated that the consideration of BMI might be useful in the treatment of elderly diabetic patients with CKD. Considering that DM in the elderly is metabolically different from DM in younger people [30], DM can be reasonably used as an attribute for cross classification. 
Kidney
Blood Pressure

Research
Kidney Blood Press Res 2019;44:362-383

DOI: 10.1159/000501021

www.karger.com/kbr

Kataoka et al.: BMI-Based Cross-Classification Approach for CKD

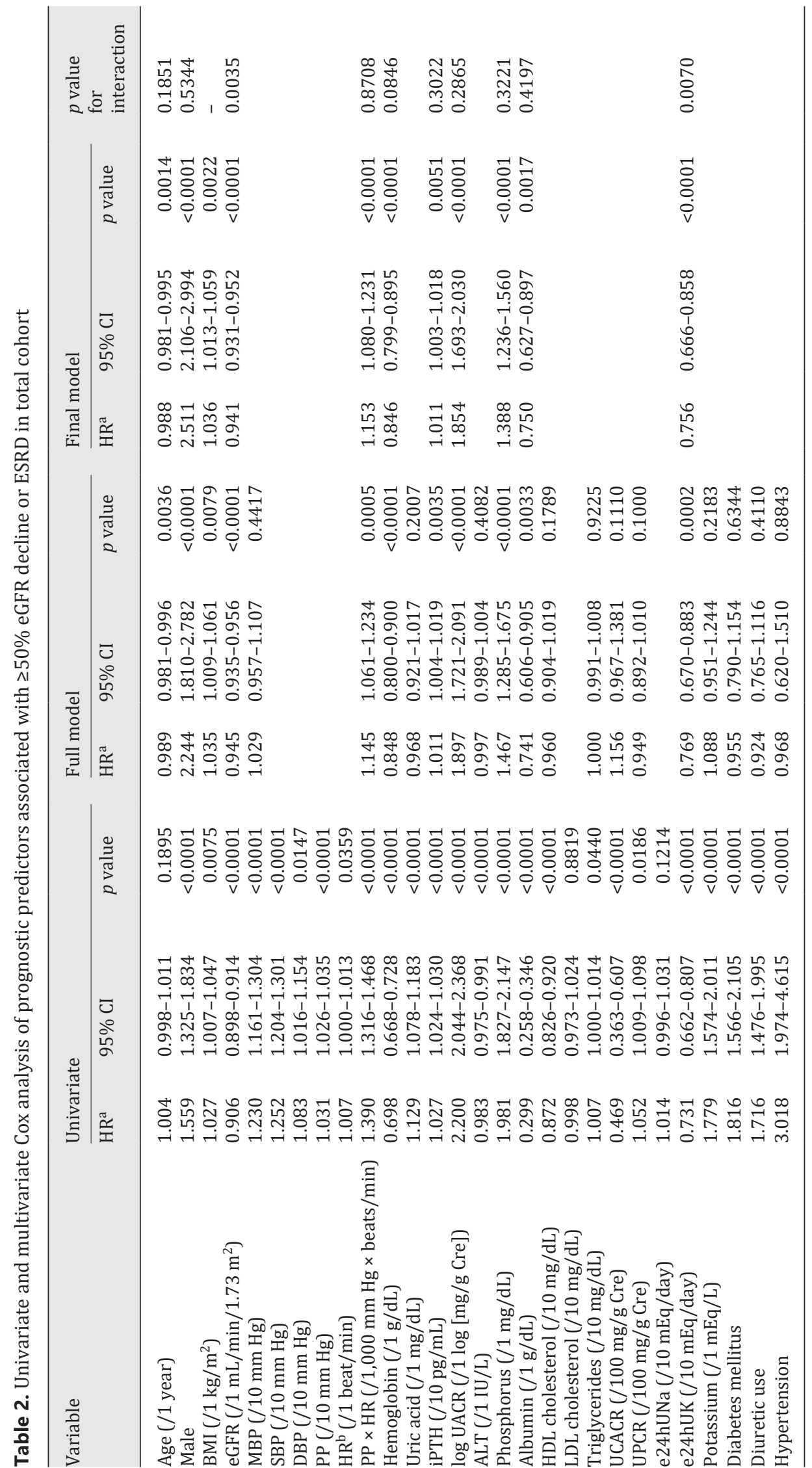

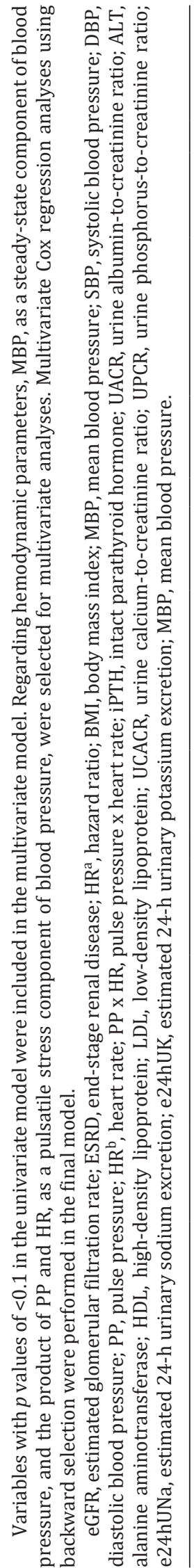




\section{Kidney \\ Blood Pressure \\ Research}

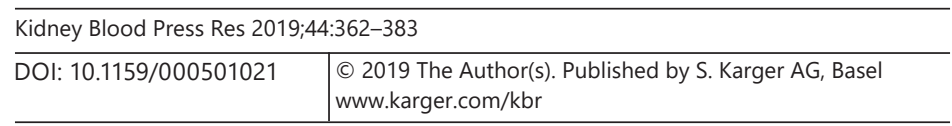

Kataoka et al.: BMI-Based Cross-Classification Approach for CKD

In the present study, we investigated many clinical factors, including urinary electrolytes, to identify potentially new renal prognostic factors using multivariate Cox regression analysis (Table 2).

Among the identified prognostic factors in the present study, albuminuria [31], kidney impairment [24, 31-34], male sex [21-24, 34-36], hyperphosphatemia [37-42], anemia [31, 32, $37,43,44]$, hypoalbuminemia [31], overweight/obesity [45, 46], younger age [32, 34], increased iPTH [47], and decreased urinary potassium excretion [48-50] have been previously reported as renal prognostic factors for CKD $[21,51,52]$. However, there are no reports using a BMI-based cross-classification approach for the assessment of prognostic factors. The present study provides additional findings regarding previous prognostic factors by using BMI-based cross classification. Although albuminuria, kidney impairment, male sex, hyperphosphatemia, anemia, and PP $\times$ HR were stable renal prognostic factors in almost all subcohorts, the prognostic value of having overweight/obese status, younger age, hypoalbuminemia, increased iPTH, and decreased urinary potassium excretion differed according to subcohort. Among these identified prognostic factors, the present study is the first to show that $\mathrm{PP} \times \mathrm{HR}$ (pulsatile stress) is a prognostic factor for CKD progression. Furthermore, we found that decreased urinary potassium excretion is a risk factor for CKD progression in patients with both $\mathrm{BMI}<25$ and CKD stages 4-5.

$\mathrm{PP} \times \mathrm{HR}$ was independently associated with CKD progression, not only in the total cohort but also in many subcohorts. Especially, these results are clinically important for the "BMI $<25$, CKD stage 3" subcohort (Fig. 14a), as the number of identified renal prognostic factors was less for this subcohort. Recently, PP was found to be associated with CKD progression for all CKD stages [53]. In a review by Safar [54], PP $\times$ HR was said to be significant, in that HR has more substantial links with PP than with mean blood pressure; an increase in PP $\times \mathrm{HR}$, more than PP itself, might cause vascular wall fatigue, particularly in the presence of atherosclerosis. Furthermore, $\mathrm{PP} \times \mathrm{HR}$ is associated with a higher risk of albuminuria in renal transplant recipients [55]. Although further investigation is needed, $\mathrm{PP} \times \mathrm{HR}$ is expected to receive more attention as an important parameter in clinical settings.

The current results may have broader worldwide implications for patients with various diseases. The BMI-based cross-classification analysis performed in the present study is consistent with treatment according to the patient's body size in Oriental medicine, suggesting a link between Oriental and Western medicine. The present study's strengths lie in the detailed analyses, designed to disaggregate data using BMI-based cross classification, which is significant to achieving PCM. We found differences in the predictive characteristics and cutoff values of several risk factors for predicting renal outcomes, via an analysis of crossclassified subcohorts. Despite these strengths, our study had several limitations. First, the patient characteristics were determined only at baseline, and data during the follow-up period were not considered. Second, the study was observational in nature; thus, any observed associations do not prove causality. Third, all of the patients in the present study were outpatients under nephrology care at major regional medical institutions; thus, a selection bias cannot be ruled out. Fourth, we did not investigate dietary factors, which may influence urinary potassium excretion. Fifth, the sample size was relatively small for a cross-classification analysis and prevented us from performing cross-classification analyses using $>2$ attributes simultaneously. Further studies are needed to confirm our findings in a larger cohort.

\section{Conclusion}

A BMI-based cross-classification approach, which provides more comprehensive findings than that in previous approaches, is expected to be an effective method for evaluating patients with CKD who are affected by multiple risk factors. 


\section{Acknowledgments}

We express our gratitude to Dr. Takahiro Mochizuki (Deceased June 25, 2017) for his advice on this work and for his contribution to medical care and medical research in Japan.

This study was conducted by principal investigators at the following medical centers: Japan Community Health Care Organization Sendai Hospital (Miyagi), JA Toride Medical Center (Ibaraki), Jichi Medical University (Tochigi), Tokyo Women's Medical University Hospital (Tokyo), St. Luke's International Hospital (Tokyo), Showa University Hospital (Tokyo), Showa University Yokohama Northern Hospital (Kanagawa), Showa University Fujigaoka Hospital (Kanagawa), Gifu Prefectural General Medical Center (Gifu), Kasugai Municipal Hospital (Aichi), Tosei General Hospital (Aichi), Osaka University Hospital (Osaka), Osaka General Medical Center (Osaka), Osaka City General Hospital (Osaka), Kurashiki Central Hospital (Okayama), Fukuoka Red Cross Hospital (Fukuoka), and Iizuka Hospital (Fukuoka).

\section{Statement of Ethics}

All procedures performed in studies involving human participants were in accordance with the ethical standards of the institutional and/or national research committee at which the studies were conducted (Tokyo Women's Medical University: \#1106) and with the 1964 Helsinki Declaration and its later amendments or comparable ethical standards. Informed consent was obtained from all individual participants included in the study.

\section{Disclosure Statement}

H.K. and T.M.: belong to the endowed chairs associated with Kyowa Hakko Kirin Co., Ltd. K.O.: has no conflicts to declare. N.H.: has served as a scientific adviser for Kyowa Hakko Kirin Co., Ltd., and received a lecture fee from the company. K.N.: has consulted for and received a research support grant from Kyowa Hakko Kirin Co., Ltd. E.I.: has consulted for Kyowa Hakko Kirin Co., Ltd. A.H.: received a research support grant from Kyowa Hakko Kirin Co., Ltd.

\section{Funding Sources}

The present study was supported by research funds with no restriction on publication from Kyowa Hakko Kirin Co., Ltd.

\section{Author Contributions}

H.K., K.O., and K.N.: were involved in study conception and design. K.O.: extracted the data. H.K. and K.O.: completed the data analysis. T.M., N.H., E.I., A.H., and K.N.: interpreted the data and provided important intellectual input. H.K. and K.O.: wrote the first draft. T.M., N.H., E.I., A.H., and K.N.: read and commented on the manuscript.

\section{References}

1 Hsu CY, Iribarren C, McCulloch CE, Darbinian J, Go AS. Risk factors for end-stage renal disease: 25-year followup. Arch Intern Med. 2009 Feb;169(4):342-50.

2 Kataoka H, Ohara M, Shibui K, Sato M, Suzuki T, Amemiya N, et al. Overweight and obesity accelerate the progression of IgA nephropathy: prognostic utility of a combination of BMI and histopathological parameters. Clin Exp Nephrol. 2012 Oct;16(5):706-12.

3 Bonnet F, Deprele C, Sassolas A, Moulin P, Alamartine E, Berthezène F, et al. Excessive body weight as a new independent risk factor for clinical and pathological progression in primary IgA nephritis. Am J Kidney Dis. 2001 Apr;37(4):720-7. 


\section{Kidney \\ Blood Pressure \\ Research}

\begin{tabular}{l|l}
\hline Kidney Blood Press Res 2019;44:362-383 \\
\hline DOI: 10.1159/000501021 & $\begin{array}{l}\text { @ 2019 The Author(s). Published by S. Karger AG, Basel } \\
\text { www.karger.com/kbr }\end{array}$ \\
\hline
\end{tabular}

Kataoka et al.: BMI-Based Cross-Classification Approach for CKD

4 Ouyang Y, Xie J, Yang M, Zhang X, Ren H, Wang W, et al. Underweight Is an Independent Risk Factor for Renal Function Deterioration in Patients with IgA Nephropathy. PLoS One. 2016 Sep;11(9):e0162044.

5 Reynolds K, Gu D, Muntner P, Chen J, Wu X, Yau CL, et al. Body mass index and risk of ESRD in China. Am J Kidney Dis. 2007 Nov;50(5):754-64.

6 Lu JL, Molnar MZ, Naseer A, Mikkelsen MK, Kalantar-Zadeh K, Kovesdy CP. Association of age and BMI with kidney function and mortality: a cohort study. Lancet Diabetes Endocrinol. 2015 Sep;3(9):704-14.

7 Imai E, Matsuo S, Makino H, Watanabe T, Akizawa T, Nitta K, et al.; CKD-JAC Study Group. Chronic Kidney Disease Japan Cohort (CKD-JAC) study: design and methods. Hypertens Res. 2008 Jun;31(6):1101-7.

8 Imai E, Matsuo S, Makino H, Watanabe T, Akizawa T, Nitta K, et al. Chronic Kidney Disease Japan Cohort study: baseline characteristics and factors associated with causative diseases and renal function. Clin Exp Nephrol. 2010 Dec;14(6):558-70.

9 Matsuo S, Imai E, Horio M, Yasuda Y, Tomita K, Nitta K, et al.; Collaborators developing the Japanese equation for estimated GFR. Revised equations for estimated GFR from serum creatinine in Japan. Am J Kidney Dis. 2009 Jun;53(6):982-92.

10 Tanaka T, Okamura T, Miura K, Kadowaki T, Ueshima H, Nakagawa H, et al. A simple method to estimate populational 24-h urinary sodium and potassium excretion using a casual urine specimen. J Hum Hypertens. 2002 Feb;16(2):97-103.

11 WHO Expert Consultation. Appropriate body-mass index for Asian populations and its implications for policy and intervention strategies. Lancet. 2004 Jan;363(9403):157-63.

12 Clark TG, Altman DG. Developing a prognostic model in the presence of missing data: an ovarian cancer case study. J Clin Epidemiol. 2003 Jan;56(1):28-37.

13 White IR, Royston P. Imputing missing covariate values for the Cox model. Stat Med. 2009 Jul;28(15):1982-98.

14 Heagerty PJ, Lumley T, Pepe MS. Time-dependent ROC curves for censored survival data and a diagnostic marker. Biometrics. 2000 Jun;56(2):337-44.

15 Glas AS, Lijmer JG, Prins MH, Bonsel GJ, Bossuyt PM. The diagnostic odds ratio: a single indicator of test performance. J Clin Epidemiol. 2003 Nov;56(11):1129-35.

16 Sacristán JA. Patient-centered medicine and patient-oriented research: improving health outcomes for individual patients. BMC Med Inform Decis Mak. 2013 Jan;13(1):6.

17 Bardes CL. Defining "patient-centered medicine”. N Engl J Med. 2012 Mar;366(9):782-3.

18 Kravitz RL, Duan N, Braslow J. Evidence-based medicine, heterogeneity of treatment effects, and the trouble with averages. Milbank Q. 2004;82(4):661-87.

19 Sacristán JA. Clinical research and medical care: towards effective and complete integration. BMC Med Res Methodol. 2015 Jan;15(1):4.

20 Hayward RA, Kent DM, Vijan S, Hofer TP. Multivariable risk prediction can greatly enhance the statistical power of clinical trial subgroup analysis. BMC Med Res Methodol. 2006 Apr;6(1):18.

21 Tsai WC, Wu HY, Peng YS, Ko MJ, Wu MS, Hung KY, et al. Risk Factors for Development and Progression of Chronic Kidney Disease: A Systematic Review and Exploratory Meta-Analysis. Medicine (Baltimore). 2016 Mar;95(11):e3013.

22 Neugarten J, Acharya A, Silbiger SR. Effect of gender on the progression of nondiabetic renal disease: a metaanalysis. J Am Soc Nephrol. 2000 Feb;11(2):319-29.

23 Seliger SL, Davis C, Stehman-Breen C. Gender and the progression of renal disease. Curr Opin Nephrol Hypertens. 2001 Mar;10(2):219-25.

24 Eriksen BO, Ingebretsen OC. The progression of chronic kidney disease: a 10-year population-based study of the effects of gender and age. Kidney Int. 2006 Jan;69(2):375-82.

25 Carrero JJ. Gender differences in chronic kidney disease: underpinnings and therapeutic implications. Kidney Blood Press Res. 2010;33(5):383-92.

26 Uhlig K, Boyd C. Guidelines for the older adult with CKD. Am J Kidney Dis. 2011 Aug;58(2):162-5.

27 O'Hare AM, Choi AI, Bertenthal D, Bacchetti P, Garg AX, Kaufman JS, et al. Age affects outcomes in chronic kidney disease. J Am Soc Nephrol. 2007 Oct;18(10):2758-65.

28 Coresh J, Turin TC, Matsushita K, Sang Y, Ballew SH, Appel LJ, et al. Decline in estimated glomerular filtration rate and subsequent risk of end-stage renal disease and mortality. JAMA. 2014 Jun;311(24):2518-31.

29 Lipska KJ, Krumholz H, Soones T, Lee SJ. Polypharmacy in the Aging Patient: A Review of Glycemic Control in Older Adults With Type 2 Diabetes. JAMA. 2016 Mar;315(10):1034-45.

30 Meneilly GS, Knip A, Tessier D; Canadian Diabetes Association Clinical Practice Guidelines Expert Committee. Diabetes in the elderly. Can J Diabetes. 2013 Apr;37(Suppl 1):S184-90.

31 Keane WF, Zhang Z, Lyle PA, Cooper ME, de Zeeuw D, Grunfeld JP, et al.; RENAAL Study Investigators. Risk scores for predicting outcomes in patients with type 2 diabetes and nephropathy: the RENAAL study. Clin J Am Soc Nephrol. 2006 Jul;1(4):761-7.

32 Hoefield RA, Kalra PA, Baker P, Lane B, New JP, O'Donoghue DJ, et al. Factors associated with kidney disease progression and mortality in a referred CKD population. Am J Kidney Dis. 2010 Dec;56(6):1072-81.

33 Inaguma D, Imai E, Takeuchi A, Ohashi Y, Watanabe T, Nitta K, et al. Risk factors for CKD progression in Japanese patients: findings from the Chronic Kidney Disease Japan Cohort (CKD-JAC) study. Clin Exp Nephrol. 2017 Jun;21(3):446-56. 
34 Yang W, Xie D, Anderson AH, Joffe MM, Greene T, Teal V, et al.; CRIC Study Investigators. Association of kidney disease outcomes with risk factors for CKD: findings from the Chronic Renal Insufficiency Cohort (CRIC) study. Am J Kidney Dis. 2014 Feb;63(2):236-43.

35 Haroun MK, Jaar BG, Hoffman SC, Comstock GW, Klag MJ, Coresh J. Risk factors for chronic kidney disease: a prospective study of 23,534 men and women in Washington County, Maryland. J Am Soc Nephrol. 2003 Nov; 14(11):2934-41.

36 Coggins CH, Breyer Lewis J, Caggiula AW, Castaldo LS, Klahr S, Wang SR. Differences between women and men with chronic renal disease. Nephrol Dial Transplant. 1998 Jun;13(6):1430-7.

37 De Nicola L, Chiodini P, Zoccali C, Borrelli S, Cianciaruso B, Di Iorio B, et al.; SIN-TABLE CKD Study Group. Prognosis of CKD patients receiving outpatient nephrology care in Italy. Clin J Am Soc Nephrol. 2011 Oct;6(10): 2421-8.

38 O'Seaghdha CM, Hwang SJ, Muntner P, Melamed ML, Fox CS. Serum phosphorus predicts incident chronic kidney disease and end-stage renal disease. Nephrol Dial Transplant. 2011 Sep;26(9):2885-90.

39 Zoccali C, Ruggenenti P, Perna A, Leonardis D, Tripepi R, Tripepi G, et al.; REIN Study Group. Phosphate may promote CKD progression and attenuate renoprotective effect of ACE inhibition. J Am Soc Nephrol. 2011 Oct; 22(10):1923-30.

40 Chang WX, Xu N, Kumagai T, Shiraishi T, Kikuyama T, Omizo H, et al. The Impact of Normal Range of Serum Phosphorus on the Incidence of End-Stage Renal Disease by A Propensity Score Analysis. PLoS One. 2016 Apr; 11(4):e0154469.

41 Norris KC, Greene T, Kopple J, Lea J, Lewis J, Lipkowitz M, et al. Baseline predictors of renal disease progression in the African American Study of Hypertension and Kidney Disease. J Am Soc Nephrol. 2006 Oct;17(10):292836.

42 Sim JJ, Bhandari SK, Smith N, Chung J, Liu IL, Jacobsen SJ, et al. Phosphorus and risk of renal failure in subjects with normal renal function. Am J Med. 2013 Apr;126(4):311-8.

43 Kuriyama S, Tomonari H, Yoshida H, Hashimoto T, Kawaguchi Y, Sakai O. Reversal of anemia by erythropoietin therapy retards the progression of chronic renal failure, especially in nondiabetic patients. Nephron. 1997; $77(2): 176-85$.

44 Kasiske BL, O’Donnell MP, Cleary MP, Keane WF. Treatment of hyperlipidemia reduces glomerular injury in obese Zucker rats. Kidney Int. 1988 Mar;33(3):667-72.

45 Iseki K, Ikemiya Y, Kinjo K, Inoue T, Iseki C, Takishita S. Body mass index and the risk of development of endstage renal disease in a screened cohort. Kidney Int. 2004 May;65(5):1870-6.

46 Hallan S, de Mutsert R, Carlsen S, Dekker FW, Aasarød K, Holmen J. Obesity, smoking, and physical inactivity as risk factors for CKD: are men more vulnerable? Am J Kidney Dis. 2006 Mar;47(3):396-405.

47 Levin A, Djurdjev 0, Beaulieu M, Er L. Variability and risk factors for kidney disease progression and death following attainment of stage 4 CKD in a referred cohort. Am J Kidney Dis. 2008 Oct;52(4):661-71.

48 Smyth A, Dunkler D, Gao P, Teo KK, Yusuf S, O’Donnell MJ, et al.; ONTARGET and TRANSCEND investigators. The relationship between estimated sodium and potassium excretion and subsequent renal outcomes. Kidney Int. 2014 Dec;86(6):1205-12.

49 Araki S, Haneda M, Koya D, Kondo K, Tanaka S, Arima H, et al. Urinary Potassium Excretion and Renal and Cardiovascular Complications in Patients with Type 2 Diabetes and Normal Renal Function. Clin J Am Soc Nephrol. 2015 Dec;10(12):2152-8.

50 Nagata T, Sobajima H, Ohashi N, Hirakawa A, Katsuno T, Yasuda Y, et al. Association between 24h Urinary Sodium and Potassium Excretion and Estimated Glomerular Filtration Rate (eGFR) Decline or Death in Patients with Diabetes Mellitus and eGFR More than $30 \mathrm{ml} / \mathrm{min} / 1.73 \mathrm{~m} 2$. PLoS One. 2016 May;11(5):e0152306.

51 Yu HT. Progression of chronic renal failure. Arch Intern Med. 2003 Jun;163(12):1417-29.

52 Taal MW, Brenner BM. Predicting initiation and progression of chronic kidney disease: developing renal risk scores. Kidney Int. 2006 Nov;70(10):1694-705.

53 Garnier AS, Briet M. Arterial Stiffness and Chronic Kidney Disease. Pulse (Basel). 2016 Apr;3(3-4):229-41.

54 Safar ME. Pulse pressure, heart rate, and drug treatment of hypertension. Curr Hypertens Rep. 2004 Jun;6(3): $190-4$.

55 Baumann M, Pan CR, Roos M, von Eynatten M, Sollinger D, Lutz J, et al. Pulsatile stress correlates with (micro-) albuminuria in renal transplant recipients. Transpl Int. 2010 Mar;23(3):292-8. 